\title{
Fundamental solutions for the Dirac equation in curved spacetime and generalized Euler-Poisson-Darboux equation
}

\author{
Karen Yagdjian ${ }^{1}$ and Anahit Galstian ${ }^{2}$
}

\author{
School of Mathematical and Statistical Sciences, \\ University of Texas RGV, 1201 W. University Drive, \\ Edinburg, TX 78539, USA \\ ${ }^{1}$ e-mail: karen.yagdjian@utrgv.edu \\ 2 e-mail: anahit.galstyan@utrgv.edu
}

Abstract

We present the fundamental solutions for the spin-1/2 fields propagating in spacetimes with power type expansion/contraction and the fundamental solution of the Cauchy problem for the Dirac equation. The derivation of these fundamental solutions is based on formulas for the solutions to the generalized Euler-Poisson-Darboux equation, which are obtained by the integral transform approach.

Keywords Dirac equation · Einstein \& de Sitter model · FLRW models · non-Fuchsian equations · Euler-Poisson-Darboux equation

\section{Introduction. Main results}

The Dirac equation is one of the important equations of relativistic quantum mechanics, quantum electrodynamics, and quantum field theory. The Dirac equation and its quantization in curved spacetime are of great interest due to the role of spin- $\frac{1}{2}$ particles in astrophysics and cosmology. Recent observational confirmation of the expansion of the universe and the quantum field theory demand a detailed investigation of the solutions of the Dirac equation in curved spacetime (see, e.g., [3, 23] and bibliography therein). The construction of a quantum field theory in curved spacetime relies heavily on a detailed description of the solutions of the Dirac equation in a curved background. The standard models of Cosmology provide such backgrounds, which form a family of curved backgrounds under the name Friedmann-Lemaitre-RobertsonWalker (FLRW) models. For the FLRW spacetime with accelerated expansion/contraction, more exactly, for the de Sitter spacetime in [33] a fundamental solution of the Dirac operator and an explicit formula for the solution of the Cauchy problem are obtained. In [34] an examination of these explicit formulas gave the answer to such an interesting question in the physics of fundamental particles as a validity of the Huygens principle. It must be noted that several exact solutions of the Dirac equation in the FLRW spacetime, which is the basis of the standard cosmological model, obtained by the separation of variables approach answer to some very interesting questions of physics (see, e.g., $[1,6,12,15,16,17,20,27,36]$ and bibliography therein). On the other hand, the fundamental solutions to the Dirac operator can provide information that is impossible to obtain by numerical solutions of differential equations, as well as new solutions that are not among the exact solutions obtained by the separation of variables approach.

In the present paper we construct the fundamental solution of the Dirac operator and obtain the explicit formulas for the solution of the Cauchy problem for the FLRW spacetime with both accelerating and decelerating expansion or contraction. The spatially flat FLRW models considered in this paper have the metric tensor that, in Cartesian coordinates, is written as follows

$$
\left(g_{\mu \nu}\right)=\left(\begin{array}{cccc}
1 & 0 & 0 & 0 \\
0 & -a^{2}(t) & 0 & 0 \\
0 & 0 & -a^{2}(t) & 0 \\
0 & 0 & 0 & -a^{2}(t)
\end{array}\right), \quad \mu, \nu=0,1,2,3,
$$


where the scale factor $a(t)=a_{0} t^{\ell}, \ell \in \mathbb{R}, t>0$, and $x \in \mathbb{R}^{3}, x_{0}=t$. If $\ell<0$ the spacetime is contracting. In the case of $\ell>1$ the expansion is accelerating (with horizon), while for $0<\ell<1$ the expansion is decelerating. In the case of the Milne spacetime $[7,13,25] \ell=1$. The FLRW spacetime with the scale factors $a(t)=a_{0} t^{2 / 3}$ and $a(t)=a_{0} t^{1 / 2}$ (see, e.g., $[18,19]$ ) are modeling the matter dominated universe (Einstein \& de Sitter model [11]) and the radiation dominated universe, respectively. The Dirac equation in the spacetime with the metric tensor (1) is (see, e.g., [1])

$$
\left(i \gamma^{0} \partial_{t}+i \frac{1}{a(t)} \gamma^{1} \partial_{x_{1}}+i \frac{1}{a(t)} \gamma^{2} \partial_{x_{2}}+i \frac{1}{a(t)} \gamma^{3} \partial_{x_{3}}+i \frac{3 \dot{a}(t)}{2 a(t)} \gamma^{0}-m \mathbb{I}_{4}\right) \Psi=F,
$$

where the contravariant gamma matrices are (see, e.g., [5, p. 61])

$$
\gamma^{0}=\left(\begin{array}{cc}
\mathbb{I}_{2} & \mathbb{O}_{2} \\
\mathbb{O}_{2} & -\mathbb{I}_{2}
\end{array}\right), \quad \gamma^{k}=\left(\begin{array}{cc}
\mathbb{O}_{2} & \sigma^{k} \\
-\sigma^{k} & \mathbb{O}_{2}
\end{array}\right), \quad k=1,2,3
$$

Here $\sigma^{k}$ are the Pauli matrices

$$
\sigma^{1}=\left(\begin{array}{ll}
0 & 1 \\
1 & 0
\end{array}\right), \quad \sigma^{2}=\left(\begin{array}{cc}
0 & -i \\
i & 0
\end{array}\right), \quad \sigma^{3}=\left(\begin{array}{cc}
1 & 0 \\
0 & -1
\end{array}\right)
$$

and $\mathbb{I}_{n}, \mathbb{O}_{n}$ denote the $n \times n$ identity and zero matrices, respectively.

We consider the fundamental solutions and the Cauchy problem with the initial time $t=\varepsilon>0$. Furthermore, we admit that the mass of the field (particle) can be changing in time and vanishing at infinity. More exactly, the model that we choose is determined by the Dirac operator

$$
\mathscr{D}\left(t, \partial_{t}, \partial_{x}\right):=i \gamma^{0} \partial_{t}+i \frac{1}{a(t)} \gamma^{1} \partial_{x_{1}}+i \frac{1}{a(t)} \gamma^{2} \partial_{x_{2}}+i \frac{1}{a(t)} \gamma^{3} \partial_{x_{3}}+i \frac{3 \dot{a}(t)}{2 a(t)} \gamma^{0}-m t^{-1} \mathbb{I}_{4},
$$

where $m \in \mathbb{C}$. This model includes the equation of a neutrino with $m=0$ as well as a massive spin- $\frac{1}{2}$ particle undergoing a redshifting of its wavelength as the universe expands.

We start with the fundamental solution to the Dirac operator. Recall that a retarded fundamental solution (a retarded inverse) for the Dirac operator (3) is a four-dimensional matrix with the operator-valued entries $\mathcal{E}^{r e t}=\mathcal{E}^{r e t}\left(x, t ; x_{0}, t_{0} ; m\right)$ that solves the equation

$$
\mathscr{D}\left(t, \partial_{t}, \partial_{x}\right) \mathcal{E}\left(x, t ; x_{0}, t_{0} ; m\right)=\delta\left(x-x_{0}\right) \delta\left(t-t_{0}\right) \mathbb{I}_{4}, \quad(x, t),\left(x_{0}, t_{0}\right) \in \mathbb{R}^{3} \times \mathbb{R}_{+},
$$

and with the support in the chronological future (causal future) $D_{+}\left(x_{0}, t_{0}\right)$ of the point $\left(x_{0}, t_{0}\right) \in \mathbb{R}^{3} \times \mathbb{R}_{+}$. The advanced fundamental solution (propagator) $\mathcal{E}^{a d v}=\mathcal{E}^{a d v}\left(x, t ; x_{0}, t_{0} ; m\right)$ solves the equation (4) and has a support in the chronological past (causal past) $D_{-}\left(x_{0}, t_{0}\right)$. The forward and backward light cones are defined as the boundaries of

$$
D_{ \pm}\left(x_{0}, t_{0}\right):=\left\{(x, t) \in \mathbb{R}^{3} \times \mathbb{R}_{+} ;\left|x-x_{0}\right| \leq \pm\left(\phi(t)-\phi\left(t_{0}\right)\right)\right\}
$$

where $\phi(t):=\frac{1}{1-\ell} t^{1-\ell}$ if $\ell \neq 1$, and $\phi(t):=\ln (t+1)$ if $\ell=1$, is a distance function when $\ell<1$. In fact, any intersection of $D_{-}\left(x_{0}, t_{0}\right)$ with the hyperplane $0<t=$ const $<t_{0}$ determines the so-called dependence domain for the point $\left(x_{0}, t_{0}\right)$, while the intersection of $D_{+}\left(x_{0}, t_{0}\right)$ with the hyperplane $t=$ const $>t_{0}>0$ is the so-called domain of influence of the point $\left(x_{0}, t_{0}\right)$. The Dirac equation (2) is non-invariant with respect to time inversion and its solutions have different properties in different direction of time.

In order to distinguish upper and lower 2-spinors we introduce two more $\gamma$-matrices (projection operators), the upper-left corner and lower-right corner matrices,

$$
\gamma^{U}=\left(\begin{array}{cc}
\mathbb{I}_{2} & \mathbb{O}_{2} \\
\mathbb{O}_{2} & \mathbb{O}_{2}
\end{array}\right)=\frac{1}{2}\left(\mathbb{I}_{4}+\gamma^{0}\right), \quad \gamma^{L}=\left(\begin{array}{cc}
\mathbb{O}_{2} & \mathbb{O}_{2} \\
\mathbb{O}_{2} & \mathbb{I}_{2}
\end{array}\right)=\frac{1}{2}\left(\mathbb{I}_{4}-\gamma^{0}\right) .
$$

Next we define the right co-factor

$$
\mathscr{D}^{c o}\left(t, \partial_{t}, \partial_{x}\right):=i t^{-\frac{\ell}{2}} \gamma^{0}\left(t^{i m} \gamma^{U}+t^{-i m} \gamma^{L}\right) \frac{\partial}{\partial t}+i t^{-\frac{3 \ell}{2}} \sum_{k=1}^{3} \gamma^{k}\left(t^{i m} \gamma^{U}+t^{-i m} \gamma^{L}\right) \frac{\partial}{\partial x_{k}}
$$


of the Dirac operator $\mathscr{D}\left(t, \partial_{t}, \partial_{x}\right)$ of (3), which is chosen such that the composition $\mathscr{D}\left(t, \partial_{t}, \partial_{x}\right) \mathscr{D}^{c o}\left(t, \partial_{t}, \partial_{x}\right)$ is a diagonal matrix of operators. In fact, the complementary operator $\mathscr{D}^{c o}\left(t, \partial_{t}, \partial_{x}\right)$ is not unique. For the Dirac equation in the de Sitter spacetime such complementary operators were first suggested in [33].

Denote $\mathcal{E}^{w}(x, t)$ to be the distribution that is the fundamental solution to the Cauchy problem for the wave equation in the Minkowski spacetime

$$
\mathcal{E}_{t t}^{w}-\Delta \mathcal{E}^{w}=0, \quad \mathcal{E}^{w}(x, 0)=\delta(x), \quad \mathcal{E}_{t}^{w}(x, 0)=0 .
$$

Here $\Delta$ is the Laplace operator in $\mathbb{R}^{3}$. Henceforth, $F(\alpha, \beta ; \gamma ; z)$ is the hypergeometric function (see, e.g., $[2])$. Our first main theorem gives for the Dirac operator the fundamental solution (retarded propagator) with support in the forward cone.

Theorem 1.1 For every $x_{0} \in \mathbb{R}^{3}, t, t_{0} \in \mathbb{R}_{+}$, the fundamental solution $\mathcal{E}_{+}\left(x, t ; x_{0}, t_{0} ; m\right)$ with support in the forward cone $D_{+}\left(x_{0}, t_{0}\right)$, that is, a distribution satisfying

$$
\left\{\begin{array}{l}
\mathscr{D}\left(t, \partial_{t}, \partial_{x}\right) \mathcal{E}_{+}\left(x, t ; x_{0}, t_{0} ; m\right)=\delta\left(x-x_{0}\right) \delta\left(t-t_{0}\right) \mathbb{I}_{4} \\
\operatorname{supp} \mathcal{E}_{+} \subseteq D_{+}\left(x_{0}, t_{0}\right)
\end{array}\right.
$$

is given as follows

$$
\begin{aligned}
& \mathcal{E}_{+}\left(x, t ; x_{0}, t_{0} ; m\right) \\
= & -2 t_{0}^{\frac{\ell}{2}-i m} \mathscr{D}^{c o}\left(t, \partial_{t}, \partial_{x}\right) \int_{0}^{\phi(t)-\phi\left(t_{0}\right)}\left(\begin{array}{cc}
E\left(r, t ; t_{0} ; m\right) \mathbb{I}_{2} & \mathbb{O}_{2} \\
\mathbb{O}_{2} & E\left(r, t ; t_{0} ;-m\right) \mathbb{I}_{2}
\end{array}\right) \mathcal{E}^{w}\left(x-x_{0}, r\right) d r,
\end{aligned}
$$

where $\ell \in \mathbb{R}, \ell \neq 1$, and

$$
\begin{aligned}
E\left(r, t ; t_{0} ; m\right)= & 2^{\frac{2 i m}{1-\ell}-1}(1-\ell)^{\frac{\ell}{1-\ell}} \phi\left(t_{0}\right)^{\frac{\ell+2 i m}{1-\ell}}\left(\left(\phi(t)+\phi\left(t_{0}\right)\right)^{2}-r^{2}\right)^{-\frac{i m}{1-\ell}} \\
& \times F\left(i \frac{m}{1-\ell}, i \frac{m}{1-\ell} ; 1 ; \frac{\left(\phi(t)-\phi\left(t_{0}\right)\right)^{2}-r^{2}}{\left(\phi(t)+\phi\left(t_{0}\right)\right)^{2}-r^{2}}\right) .
\end{aligned}
$$

The fundamental solution to the Cauchy problem is given by the next theorem.

Theorem 1.2 For every positive $\varepsilon>0$ and $t>\varepsilon$ the fundamental solution $\mathcal{E}_{+}\left(x, t ; x_{0} ; m ; \varepsilon\right)$ to the Cauchy problem, that is, a distribution satisfying

$$
\left\{\begin{array}{l}
\mathscr{D}\left(t, \partial_{t}, \partial_{x}\right) \mathcal{E}_{+}\left(x, t ; x_{0} ; m ; \varepsilon\right)=\mathbb{O}_{4} \\
\mathcal{E}_{+}\left(x, \varepsilon ; x_{0} ; m ; \varepsilon\right)=\delta\left(x-x_{0}\right) \mathbb{I}_{4}
\end{array}\right.
$$

is given as follows

$$
\begin{aligned}
\mathcal{E}_{+}\left(x, t ; x_{0} ; m ; \varepsilon\right)= & -i \varepsilon^{1+\frac{\ell}{2}-i m}(1-\ell)^{-1} \mathscr{D}^{c o}\left(x, t, \partial_{t}, \partial_{x}\right) \gamma^{0} \\
& \times \int_{0}^{\phi(t)-\phi(\varepsilon)}\left(\begin{array}{cc}
K_{1}(r, t ; m ; \varepsilon) \mathbb{I}_{2} & \mathbb{O}_{2} \\
\mathbb{O}_{2} & K_{1}(r, t ;-m ; \varepsilon) \mathbb{I}_{2}
\end{array}\right) \mathcal{E}^{w}\left(x-x_{0}, r\right) d r,
\end{aligned}
$$

where $\ell \in \mathbb{R}, \ell \neq 1$, and

$$
\begin{aligned}
& K_{1}(r, t ; m ; \varepsilon) \\
:= & 2^{2 i \frac{m}{1-\ell}} \phi(\varepsilon)^{2 i \frac{m}{1-\ell}-1}\left((\phi(t)+\phi(\varepsilon))^{2}-r^{2}\right)^{-i \frac{m}{1-\ell}} F\left(i \frac{m}{1-\ell}, i \frac{m}{1-\ell} ; 1 ; \frac{(\phi(t)-\phi(\varepsilon))^{2}-r^{2}}{(\phi(t)+\phi(\varepsilon))^{2}-r^{2}}\right) .
\end{aligned}
$$

In order to write the solution to the Cauchy problem we introduce the operator

$$
\begin{aligned}
& \mathcal{G}\left(x, t, D_{x} ; m\right)[f](x, t) \\
:= & -2 \int_{\varepsilon}^{t} b^{\frac{\ell}{2}-i m} d b \int_{0}^{\phi(t)-\phi(b)} E(r, t ; b ; m) \int_{\mathbb{R}^{3}} \mathcal{E}^{w}(x-y, r) f(y, b) d y d r, \quad f \in C_{0}^{\infty}\left(\mathbb{R}^{n+1}\right),
\end{aligned}
$$


and the operator $\mathcal{K}_{1}\left(x, t, D_{x} ; m ; \varepsilon\right)$ as follows:

$$
\begin{aligned}
& \mathcal{K}_{1}\left(x, t, D_{x} ; m ; \varepsilon\right)[\varphi](x, t) \\
:= & -i \varepsilon^{1+\frac{\ell}{2}-i m}(1-\ell)^{-1} \int_{0}^{\phi(t)-\phi(\varepsilon)} K_{1}(r, t ; m ; \varepsilon) \int_{\mathbb{R}^{3}} \mathcal{E}^{w}(x-y, r) \varphi(y) d y d r, \quad \varphi \in C_{0}^{\infty}\left(\mathbb{R}^{n}\right) .
\end{aligned}
$$

Theorem 1.3 The solution to the Cauchy problem

$$
\left\{\begin{array}{l}
\mathscr{D}\left(t, \partial_{t}, \partial_{x}\right) \Psi(x, t)=F(x, t), \quad t>\varepsilon>0 \\
\Psi(x, \varepsilon)=\Psi_{\varepsilon}(x)
\end{array}\right.
$$

with $m \in \mathbb{C}$, is given as follows

$$
\begin{aligned}
\Psi(x, t)= & \mathscr{D}^{c o}\left(t, \partial_{t}, \partial_{x}\right)\left\{\left(\begin{array}{cc}
\mathcal{G}\left(x, t, D_{x} ; m\right) \mathbb{I}_{2} & \mathbb{O}_{2} \\
\mathbb{O}_{2} & \mathcal{G}\left(x, t, D_{x} ;-m\right) \mathbb{I}_{2}
\end{array}\right)[F](x, t)\right. \\
& \left.+\gamma^{0}\left(\begin{array}{cc}
\mathcal{K}_{1}\left(x, t, D_{x} ; m ; \varepsilon\right) \mathbb{I}_{2} & \mathbb{O}_{2} \\
\mathbb{O}_{2} & \mathcal{K}_{1}\left(x, t, D_{x} ;-m ; \varepsilon\right) \mathbb{I}_{2}
\end{array}\right)\left[\Psi_{\varepsilon}\right](x, t)\right\}, \quad t>\varepsilon>0 .
\end{aligned}
$$

For the case of $\ell=0$ see [33].

The derivation of the formulas for the fundamental solutions and for the solution of the Cauchy problem is carried out in three steps. The first step (Section 2) is the finding of a complementary operator that reduces the $4 \times 4$ system of the first order operators to the system of the diagonal operator-valued matrix with two pairs of the coinciding non-Fuchsian second-order hyperbolic partial differential operators. The second step (Section 4) is a reduction of such second-order hyperbolic partial differential operators to the generalized Euler-Poisson-Darboux equation

$$
\partial_{t}^{2} u-A\left(x, \partial_{x}\right) u+\frac{2 i m}{t} \partial_{t} u=f
$$

where $m \in \mathbb{C}, t \in \mathbb{R}_{+}$and $A\left(x, \partial_{x}\right)$ can be a pseudo-differential operator with the symbol $A(x, \xi)$ defined for $(x, \xi) \in \Omega \times \mathbb{R}^{n}$ and $\Omega$ is a domain in $\mathbb{R}^{n}$. The reduction is done by a change to the co-moving coordinates.

The last, the third step (Section 5), is devoted to solving the Cauchy problem for the generalized EulerPoisson-Darboux equation (10) (Theorems 5.3-5.5). In the equation (10) higher order derivatives with respect to spatial variables may appear. To solve the last equation the integral transform approach from $[30,31,32,35]$ is employed. In the last two steps it is supposed that $x \in \mathbb{R}^{n}$ with an arbitrary dimension $n$.

\section{The complementary operator}

The non-Fuchsian Type Dirac operator $\mathscr{D}\left(t, \partial_{t}, \partial_{x}\right)$ in the FLRW spacetime is defined by

$$
\mathscr{D}\left(t, \partial_{t}, \partial_{x}\right):=i \gamma^{0} \frac{\partial}{\partial t}+i t^{-\ell} \gamma^{1} \frac{\partial}{\partial x_{1}}+i t^{-\ell} \gamma^{2} \frac{\partial}{\partial x_{2}}+i t^{-\ell} \gamma^{3} \frac{\partial}{\partial x_{3}}+i \frac{3}{2} \ell t^{-1} \gamma^{0}-m t^{-1} \mathbb{I}_{4} .
$$

With the aid of the matrices $\gamma^{U}$ and $\gamma^{L}(5)$ we calculate

$$
\begin{aligned}
& t^{c} \gamma^{U}+\gamma^{L}=\left(\begin{array}{cc}
t^{c} \mathbb{I}_{2} & \mathbb{O}_{2} \\
\mathbb{O}_{2} & \mathbb{I}_{2}
\end{array}\right), \quad\left(t^{c} \gamma^{U}+\gamma^{L}\right) \gamma^{0}\left(t^{r} \gamma^{U}+\gamma^{L}\right)=\left(\begin{array}{cc}
t^{c+r} \mathbb{I}_{2} & \mathbb{O}_{2} \\
\mathbb{O}_{2} & -\mathbb{I}_{2}
\end{array}\right), \\
& \left(t^{c} \gamma^{U}+\gamma^{L}\right) \gamma^{k}\left(t^{r} \gamma^{U}+\gamma^{L}\right)=\left(\begin{array}{cc}
\mathbb{O}_{2} & t^{c} \sigma^{k} \\
-t^{r} \sigma^{k} & \mathbb{O}_{2}
\end{array}\right), \quad k=1,2,3 .
\end{aligned}
$$

Next we use them to define a family of complementary factors $\mathscr{D}^{c o}\left(t, \partial_{t}, \partial_{x}\right)$. For the Dirac operator the auxiliary right complementary factors to the diagonal matrix with the operator-valued entries form a family of operators, which contains the following operators

$$
\begin{aligned}
\mathscr{D}^{c o}\left(t, \partial_{t}, \partial_{x}\right):= & i t^{b}\left(t^{c} \gamma^{U}+\gamma^{L}\right) \gamma^{0} \frac{\partial}{\partial t}\left(t^{r} \gamma^{U}+\gamma^{L}\right)+i t^{-\ell} t^{b}\left(t^{c} \gamma^{U}+\gamma^{L}\right) \gamma^{1}\left(t^{r} \gamma^{U}+\gamma^{L}\right) \frac{\partial}{\partial x_{1}}+ \\
& +i t^{-\ell} t^{b}\left(t^{c} \gamma^{U}+\gamma^{L}\right) \gamma^{2}\left(t^{r} \gamma^{U}+\gamma^{L}\right) \frac{\partial}{\partial x_{2}}+i t^{-\ell} t^{b}\left(t^{c} \gamma^{U}+\gamma^{L}\right) \gamma^{3}\left(t^{r} \gamma^{U}+\gamma^{L}\right) \frac{\partial}{\partial x_{3}}+ \\
& +i \frac{3}{4} b_{0} t^{b-1}\left(t^{c} \gamma^{U}+\gamma^{L}\right) \gamma^{0}\left(t^{r} \gamma^{U}+\gamma^{L}\right)+n t^{b-1}\left(t^{c} \gamma^{U}+\gamma^{L}\right)\left(t^{r} \gamma^{U}+\gamma^{L}\right) .
\end{aligned}
$$


These operators depend on parameters $b_{0}, b, c, n, r \in \mathbb{C}$. We need also a matrix

$$
t^{a} \gamma^{U}+t^{\omega} \gamma^{L}=\left(\begin{array}{cc}
t^{a} \mathbb{I}_{2} & \mathbb{O}_{2} \\
\mathbb{O}_{2} & t^{\omega} \mathbb{I}_{2}
\end{array}\right)
$$

Then for the parameters $a, b_{0}, c, n$ chosen as follows

$$
a:=\frac{\ell}{2}-i m, \quad \omega:=\frac{\ell}{2}+i m, \quad b_{0}=-\frac{4 i m}{3}, \quad b=-i m-\frac{\ell}{2}, \quad r=2 i m, \quad c=0, \quad n=m,
$$

since $\gamma^{U}+\gamma^{L}=\mathbb{I}_{4}$, we obtain the operator of (6) and the following identity

$$
\begin{aligned}
& \mathscr{D}\left(t, \partial_{t}, \partial_{x}\right) \mathscr{D}^{c o}\left(t, \partial_{t}, \partial_{x}\right) \\
& =\left(t^{-a} \gamma^{U}+t^{-\omega} \gamma^{L}\right)\left(\begin{array}{cc}
-\mathbb{I}_{2}\left(\partial_{t}^{2}-t^{-2 \ell} \ell+\frac{(\ell+2 i m)}{t} \partial_{t}\right) & \mathbb{O}_{2} \\
\mathbb{O}_{2} & -\mathbb{I}_{2}\left(\partial_{t}^{2}-t^{-2 \ell} \ell+\frac{(\ell-2 i m)}{t} \partial_{t}\right)
\end{array}\right) \\
& =-\left(t^{-\frac{\ell}{2}+i m} \gamma^{U}+t^{-\frac{\ell}{2}-i m} \gamma^{L}\right)\left[\mathbb{I}_{4} \partial_{t}^{2}-t^{-2 \ell} \mathbb{I}_{4} \ell+t^{-1}\left((\ell+2 i m) \gamma^{U}+(\ell-2 i m) \gamma^{L}\right) \partial_{t}\right] .
\end{aligned}
$$

If we denote the second-order scalar hyperbolic operator

$$
P\left(t, \partial_{t}, \partial_{x} ; m\right):=\partial_{t}^{2}-t^{-2 \ell} \Delta+t^{-1}(\ell+2 i m) \partial_{t},
$$

then we obtain the identity with the diagonal right-hand side

$$
\begin{aligned}
\mathscr{D}\left(t, \partial_{t}, \partial_{x}\right) \mathscr{D}^{c o}\left(t, \partial_{t}, \partial_{x}\right) & =-\left(t^{-a} \gamma^{U}+t^{-\omega} \gamma^{L}\right)\left(\gamma^{U} P\left(t, \partial_{t}, \partial_{x} ; m\right)+\gamma^{L} P\left(t, \partial_{t}, \partial_{x} ;-m\right)\right) \\
& =-\left(\begin{array}{cc}
t^{-a} \mathbb{I}_{2} P\left(t, \partial_{t}, \partial_{x} ; m\right) & \mathbb{O}_{2} \\
\mathbb{O}_{2} & t^{-\omega} \mathbb{I}_{2} P\left(t, \partial_{t}, \partial_{x} ;-m\right)
\end{array}\right) .
\end{aligned}
$$

The complementary operator $\mathscr{D}^{c o}\left(t, \partial_{t}, \partial_{x}\right)$ is not unique. An advantage of the choice of parameters $b_{0}, b, c$, $n, r$ (11) is that the diagonal entries of (13) are the scalar operators: two operators $t^{-a} P\left(t, \partial_{t}, \partial_{x} ; m\right)$ and two operators $t^{-\omega} P\left(t, \partial_{t}, \partial_{x} ;-m\right)$, which differ only by the sign of the mass $m$ and the factors $t^{-a}$ and $t^{-\omega}$.

In order to consider the Dirac equation in curvilinear coordinates as well as to enlarge class of the partial differential equations, we follow the arguments which have been specified in [33, Sec1]. More exactly, consider operator

$$
\mathscr{D}\left(x, t, \partial_{t}, \partial_{x}\right):=i \gamma^{0} \frac{\partial}{\partial t}+i t^{-\ell} \sum_{k=1}^{3} \gamma^{k} A_{k}\left(x, \partial_{x}\right)+i \frac{3}{2} \ell t^{-1} \gamma^{0}-m t^{-1} \mathbb{I}_{4},
$$

where the operators $A_{k}\left(x, \partial_{x}\right), k=1,2,3$, in general, are the scalar pseudo-differential operators $A_{k}\left(x, \partial_{x}\right)$ with symbols depending on the spatial variables too. Following [33] we define the generalized Dirac operator (14) as an operator satisfying the condition

$$
\sum_{k, j=1,2,3}\left(t^{-i m} \gamma^{U}+t^{i m} \gamma^{L}\right) \gamma^{k} \gamma^{j}\left(t^{i m} \gamma^{U}+t^{-i m} \gamma^{L}\right) A_{k}(x, \partial) A_{j}(x, \partial)=-\mathcal{A}\left(x, \partial_{x} ; m\right) \mathbb{I}_{4} \quad \text { for all } t>0,
$$

where $\mathcal{A}\left(x, \partial_{x} ; m\right)$ is the scalar pseudo-differential operator independent of $t$. For $m=0$ this condition coincides with condition (1.17) [33]. We note that for the pairwise commuting operators this condition is satisfied with $\mathcal{A}\left(x, \partial_{x} ; m\right)=-\sum_{k=1,2,3} A_{k}^{2}(x, \partial)$. From now on we omit $m$ in the notation $\mathcal{A}\left(x, \partial_{x} ; m\right)$ and write just $\mathcal{A}\left(x, \partial_{x}\right)$.

The complementary operator $\mathscr{D}^{c o}\left(x, t, \partial_{t}, \partial_{x}\right)$ for operator (14) is given by the following theorem.

Theorem 2.1 Assume that for operator (14) condition (15) is fulfilled. Then the following operator

$$
\mathscr{D}^{c o}\left(x, t, \partial_{t}, \partial_{x}\right):=i t^{-\frac{\ell}{2}} \gamma^{0}\left(t^{i m} \gamma^{U}+t^{-i m} \gamma^{L}\right) \frac{\partial}{\partial t}+i t^{-\frac{3 \ell}{2}} \sum_{k=1}^{3} \gamma^{k}\left(t^{i m} \gamma^{U}+t^{-i m} \gamma^{L}\right) A_{k}(x, \partial)
$$

is a complementary operator $\mathscr{D}^{c o}\left(x, t, \partial_{t}, \partial_{x}\right)$ for (14), that is,

$$
\begin{aligned}
& \mathscr{D}\left(x, t, \partial_{t}, \partial_{x}\right) \mathscr{D}^{c o}\left(x, t, \partial_{t}, \partial_{x}\right) \\
= & -t^{-\frac{\ell}{2}}\left(t^{i m} \gamma^{U}+t^{-i m} \gamma^{L}\right)\left\{\mathbb{I}_{4} \frac{\partial^{2}}{\partial t^{2}}-t^{-2 \ell} \mathcal{A}\left(x, \partial_{x}\right) \mathbb{I}_{4}+t^{-1}\left(\ell \mathbb{I}_{4}+2 i m \gamma^{0}\right) \frac{\partial}{\partial t}\right\} .
\end{aligned}
$$


Proof. Indeed, it is easy to see that

$$
\mathscr{D}\left(x, t, \partial_{t}, \partial_{x}\right) \mathscr{D}^{c o}\left(x, t, \partial_{t}, \partial_{x}\right)=I_{1}+I_{2}+I_{3}+I_{4},
$$

where we have denoted

$$
\begin{aligned}
I_{1}= & i \gamma^{0} \frac{\partial}{\partial t}\left\{i t^{-\frac{\ell}{2}} \gamma^{0}\left(t^{i m} \gamma^{U}+t^{-i m} \gamma^{L}\right) \frac{\partial}{\partial t}+i t^{-\frac{3 \ell}{2}} \sum_{b=1}^{3} \gamma^{b}\left(t^{i m} \gamma^{U}+t^{-i m} \gamma^{L}\right) A_{b}(x, \partial)\right\}, \\
I_{2}= & i t^{-\ell} \sum_{a=1}^{3} \gamma^{a} A_{a}(x, \partial)\left\{i t^{-\frac{\ell}{2}} \gamma^{0}\left(t^{i m} \gamma^{U}+t^{-i m} \gamma^{L}\right) \frac{\partial}{\partial t}\right. \\
& \left.+i t^{-\frac{3 \ell}{2}} \sum_{b=1}^{3} \gamma^{b}\left(t^{i m} \gamma^{U}+t^{-i m} \gamma^{L}\right) A_{b}(x, \partial)\right\}, \\
I_{3}= & i \frac{3}{2} \ell t^{-1} i t^{-\frac{\ell}{2}}\left(t^{i m} \gamma^{U}+t^{-i m} \gamma^{L}\right) \frac{\partial}{\partial t}+i \frac{3}{2} \ell t^{-1} i t^{-\frac{3 \ell}{2}} \sum_{b=1}^{3} \gamma^{0} \gamma^{b}\left(t^{i m} \gamma^{U}+t^{-i m} \gamma^{L}\right) A_{b}(x, \partial), \\
I_{4}= & -m t^{-1} i t^{-\frac{\ell}{2}} \gamma^{0}\left(t^{i m} \gamma^{U}+t^{-i m} \gamma^{L}\right) \frac{\partial}{\partial t}-m t^{-1} i t^{-\frac{3 \ell}{2}} \sum_{b=1}^{3} \gamma^{b}\left(t^{i m} \gamma^{U}+t^{-i m} \gamma^{L}\right) A_{b}(x, \partial) .
\end{aligned}
$$

Then it is easy to derive

$$
\begin{aligned}
& I_{1}=-t^{-\frac{\ell}{2}}\left(t^{i m} \gamma^{U}+t^{-i m} \gamma^{L}\right) \frac{\partial^{2}}{\partial t^{2}}-t^{-\frac{3 \ell}{2}} \sum_{b=1}^{3} \gamma^{0} \gamma^{b}\left(t^{i m} \gamma^{U}+t^{-i m} \gamma^{L}\right) A_{b}(x, \partial) \frac{\partial}{\partial t} \\
& +\frac{\ell}{2} t^{-\frac{\ell}{2}-1}\left(t^{i m} \gamma^{U}+t^{-i m} \gamma^{L}\right) \frac{\partial}{\partial t}-i m t^{-\frac{\ell}{2}-1}\left(t^{i m} \gamma^{U}-t^{-i m} \gamma^{L}\right) \frac{\partial}{\partial t} \\
& +\frac{3 \ell}{2} t^{-\frac{3 \ell}{2}-1} \sum_{b=1}^{3} \gamma^{0} \gamma^{b}\left(t^{i m} \gamma^{U}+t^{-i m} \gamma^{L}\right) A_{b}(x, \partial) \\
& -i m t^{-\frac{3 \ell}{2}-1} \sum_{b=1}^{3} \gamma^{0} \gamma^{b}\left(t^{i m} \gamma^{U}-t^{-i m} \gamma^{L}\right) A_{b}(x, \partial) \\
& I_{2}=-t^{-\frac{3 \ell}{2}} \sum_{a=1}^{3} \gamma^{a} \gamma^{0}\left(t^{i m} \gamma^{U}+t^{-i m} \gamma^{L}\right) \frac{\partial}{\partial t} A_{a}(x, \partial) \\
& -t^{-\frac{5 \ell}{2}} \sum_{a=1}^{3} \sum_{b=1}^{3} \gamma^{a} \gamma^{b}\left(t^{i m} \gamma^{U}+t^{-i m} \gamma^{L}\right) A_{a}(x, \partial) A_{b}(x, \partial) \\
& I_{3}=-\frac{3}{2} \ell t^{-1-\frac{\ell}{2}}\left(t^{i m} \gamma^{U}+t^{-i m} \gamma^{L}\right) \frac{\partial}{\partial t}-\frac{3}{2} \ell t^{-1-\frac{3 \ell}{2}} \sum_{b=1}^{3} \gamma^{0} \gamma^{b}\left(t^{i m} \gamma^{U}+t^{-i m} \gamma^{L}\right) A_{b}(x, \partial) \text {, } \\
& I_{4}=-i m t^{-1-\frac{\ell}{2}} \gamma^{0}\left(t^{i m} \gamma^{U}+t^{-i m} \gamma^{L}\right) \frac{\partial}{\partial t}-i m t^{-1-\frac{3 \ell}{2}} \sum_{b=1}^{3} \gamma^{b}\left(t^{i m} \gamma^{U}+t^{-i m} \gamma^{L}\right) A_{b}(x, \partial) \text {. }
\end{aligned}
$$

After substitution of all these expressions in $\mathscr{D}\left(x, t, \partial_{t}, \partial_{x}\right) \mathscr{D}^{c o}\left(x, t, \partial_{t}, \partial_{x}\right)$ we obtain operator with the coefficients as follows. The coefficient of $\partial^{2} / \partial t^{2}$ is

$$
-t^{-\frac{\ell}{2}}\left(t^{i m} \gamma^{U}+t^{-i m} \gamma^{L}\right)
$$

The coefficient of the operator $\partial / \partial t$ is

$$
\begin{aligned}
& \frac{\ell}{2} t^{-\frac{\ell}{2}-1}\left(t^{i m} \gamma^{U}+t^{-i m} \gamma^{L}\right)-i m t^{-\frac{\ell}{2}-1}\left(t^{i m} \gamma^{U}-t^{-i m} \gamma^{L}\right) \\
& -\frac{3}{2} \ell t^{-1-\frac{\ell}{2}}\left(t^{i m} \gamma^{U}+t^{-i m} \gamma^{L}\right)-i m t^{-1-\frac{\ell}{2}} \gamma^{0}\left(t^{i m} \gamma^{U}+t^{-i m} \gamma^{L}\right) \\
= & t^{-\frac{\ell}{2}-1}\left(t^{i m} \gamma^{U}+t^{-i m} \gamma^{L}\right)\left(-\ell-2 i m \gamma^{0}\right) .
\end{aligned}
$$


For the terms with $A_{a}(x, \partial) \frac{\partial}{\partial t}$ we obtain

$$
\begin{aligned}
& -t^{-\frac{3 \ell}{2}} \sum_{b=1}^{3} \gamma^{0} \gamma^{b}\left(t^{i m} \gamma^{U}+t^{-i m} \gamma^{L}\right) A_{b}(x, \partial) \frac{\partial}{\partial t} \\
& -t^{-\frac{3 \ell}{2}} \sum_{a=1}^{3} \gamma^{a} \gamma^{0}\left(t^{i m} \gamma^{U}+t^{-i m} \gamma^{L}\right) A_{a}(x, \partial) \frac{\partial}{\partial t}=0 .
\end{aligned}
$$

For the terms with $A_{a}(x, \partial)$ we obtain

$$
\begin{aligned}
& \frac{3 \ell}{2} t^{-\frac{3 \ell}{2}-1} \sum_{b=1}^{3} \gamma^{0} \gamma^{b}\left(t^{i m} \gamma^{U}+t^{-i m} \gamma^{L}\right) A_{b}(x, \partial)-i m t^{-\frac{3 \ell}{2}-1} \sum_{b=1}^{3} \gamma^{0} \gamma^{b}\left(t^{i m} \gamma^{U}-t^{-i m} \gamma^{L}\right) A_{b}(x, \partial) \\
& -\frac{3}{2} \ell t^{-\frac{3 \ell}{2}-1} \sum_{b=1}^{3} \gamma^{0} \gamma^{b}\left(t^{i m} \gamma^{U}+t^{-i m} \gamma^{L}\right) A_{b}(x, \partial)-i m t^{-\frac{3 \ell}{2}-1} \sum_{b=1}^{3} \gamma^{b}\left(t^{i m} \gamma^{U}+t^{-i m} \gamma^{L}\right) A_{b}(x, \partial) \\
& =0 .
\end{aligned}
$$

The remaining terms are

$$
\begin{aligned}
& -t^{-\frac{5 \ell}{2}} \sum_{a=1}^{3} \sum_{b=1}^{3} \gamma^{a} \gamma^{b}\left(t^{i m} \gamma^{U}+t^{-i m} \gamma^{L}\right) A_{a}(x, \partial) A_{b}(x, \partial) \\
= & -\left(t^{i m} \gamma^{U}+t^{-i m} \gamma^{L}\right)\left(t^{-i m} \gamma^{U}+t^{i m} \gamma^{L}\right) t^{-\frac{5 \ell}{2}} \sum_{a=1}^{3} \gamma^{a} \gamma^{b}\left(t^{i m} \gamma^{U}+t^{-i m} \gamma^{L}\right) A_{a}(x, \partial) A_{b}(x, \partial) \\
= & t^{-\frac{5 \ell}{2}}\left(t^{i m} \gamma^{U}+t^{-i m} \gamma^{L}\right) \mathcal{A}\left(x, \partial_{x}\right) \mathbb{I}_{4} .
\end{aligned}
$$

Theorem is proved.

Remark 2.2 More general choice of the family of operators has been suggested in [33, Sec.1], which in the case of $a(t)=a_{0} t^{\ell}$ leads to

$$
\begin{aligned}
& \sum_{k, j=1,2,3}\left(t^{-i m} \gamma^{U}+t^{i m} \gamma^{L}\right) \gamma^{k} \gamma^{j}\left(t^{i m} \gamma^{U}+t^{-i m} \gamma^{L}\right) A_{k}(x, \partial) A_{j}(x, \partial) \\
= & -\mathcal{A}\left(x, \partial_{x} ; m\right) \mathbb{I}_{4}+\mathcal{B}\left(x, \partial_{x} ; m\right) \gamma^{0}+\mathcal{C}\left(x, \partial_{x} ; m\right) \gamma^{1} \gamma^{2}+\mathcal{D}\left(x, \partial_{x} ; m\right) \gamma^{3} \gamma^{0} \gamma^{1} \gamma^{2} \gamma^{3} \quad \forall t>0,
\end{aligned}
$$

where $A_{k}\left(x, \partial_{x}\right), \mathcal{A}\left(x, \partial_{x} ; m\right), \mathcal{B}\left(x, \partial_{x} ; m\right), \mathcal{C}\left(x, \partial_{x} ; m\right)$, and $\mathcal{D}\left(x, \partial_{x} ; m\right)$ are scalar pseudo-differential operators. For the purpose of the present paper it suffices to consider the case of $(15)$, that is, $\mathcal{B}\left(x, \partial_{x} ; m\right)=$ $\mathcal{C}\left(x, \partial_{x} ; m\right)=\mathcal{D}\left(x, \partial_{x} ; m\right)=0$.

In order to find a complementary operator for the Dirac operator in the FLRW spacetime in the curvilinear coordinates, we appeal to the spherical coordinates

$$
x(r, \theta, \phi):=r \cos (\phi) \sin (\theta), \quad y(r, \theta, \phi):=r \sin (\phi) \sin (\theta), \quad z(r, \theta, \phi):=r \cos (\theta)
$$

and choose a family of operators with the property (15) containing the operators with coefficients

$$
\begin{aligned}
A_{1}(x, \partial)= & \cos (\phi) \sin (\theta) \partial_{r}+\frac{\cos (\phi) \cos (\theta)}{r} \partial_{\theta}-\frac{\sin (\phi)}{r \sin (\theta)} \partial_{\phi}, \\
A_{2}(x, \partial)= & \sin (\phi) \sin (\theta) \partial_{r}+\frac{\sin (\phi) \cos (\theta)}{r} \partial_{\theta}+\frac{\cos (\phi)}{r \sin (\theta)} \partial_{\phi}, \\
A_{3}(x, \partial)= & \cos (\theta)\left(\partial_{r}+V(r)\right)-\frac{\sin (\theta)}{r} \partial_{\theta}, \\
\mathcal{A}\left(x, \partial_{x}\right)= & \partial_{r}^{2}+2 \frac{1}{r}(1+r V(r)) \partial_{r}+\frac{1}{r}\left(r V^{\prime}(r)+2 V(r)+r V^{2}(r)\right) \\
& +\frac{1}{r^{2}}\left(\partial_{\theta}^{2}+\cot (\theta) \partial_{\theta}+\csc ^{2}(\theta) \partial_{\phi}^{2}\right) .
\end{aligned}
$$


Here $x=(r, \theta, \phi)$ and $V=V(r)$ is a smooth function defined in $\mathbb{R}_{+}$.

We can write the Dirac equation (2) as follows

$$
\left(i \gamma^{0} \partial_{0}+i \frac{1}{a(t)}\left(\gamma_{c}^{r} \partial_{r}+\gamma_{c}^{\theta} \partial_{\theta}+\gamma_{c}^{\phi} \partial_{\phi}\right)+i \frac{3 \dot{a}(t)}{2 a(t)} \gamma^{0}-m t^{-1} \mathbb{I}_{4}\right) \Psi(x, t)=F(x, t),
$$

where in this Cartesian tetrad gauge the gamma matrices will be given by (see, e.g., [24])

$$
\begin{aligned}
\gamma_{c}^{r} & =\gamma^{1} \cos (\phi) \sin (\theta)+\gamma^{2} \sin (\theta) \sin (\phi)+\gamma^{3} \cos (\theta) \\
\gamma_{c}^{\phi} & =-\gamma^{1} \frac{\sin (\phi)}{r \sin (\theta)}+\gamma^{2} \frac{\cos (\phi)}{r \sin (\theta)}=\frac{1}{r \sin (\theta)}\left(-\gamma^{1} \sin (\phi)+\gamma^{2} \cos (\phi)\right) \\
\gamma_{c}^{\theta} & =\frac{1}{r}\left(\gamma^{1} \cos (\theta) \cos (\phi)+\gamma^{2} \sin (\phi) \cos (\theta)-\gamma^{3} \sin (\theta)\right) .
\end{aligned}
$$

We have used the subscript $c$ for Cartesian. We can also write

$$
\left(i \gamma^{0} \partial_{0}+i \frac{1}{a(t)}\left(\tilde{\gamma}_{c}^{r} \partial_{r}+\tilde{\gamma}_{c}^{\phi} \frac{1}{r \sin (\theta)} \partial_{\phi}+\tilde{\gamma}_{c}^{\theta} \frac{1}{r} \partial_{\theta}\right)+i \frac{3 \dot{a}(t)}{2 a(t)} \gamma^{0}-m t^{-1} \mathbb{I}_{4}\right) \Psi(x, t)=F(x, t),
$$

where $\tilde{\gamma}_{c}^{t}=\gamma^{0}$ and

$$
\begin{aligned}
& \tilde{\gamma}_{c}^{r}=\gamma_{c}^{r}=\gamma^{1} \cos (\phi) \sin (\theta)+\gamma^{2} \sin (\theta) \sin (\phi)+\gamma^{3} \cos (\theta), \\
& \tilde{\gamma}_{c}^{\phi}=-\gamma^{1} \sin (\phi)+\gamma^{2} \cos (\phi), \\
& \tilde{\gamma}_{c}^{\theta}=\gamma^{1} \cos (\theta) \cos (\phi)+\gamma^{2} \sin (\phi) \cos (\theta)-\gamma^{3} \sin (\theta),
\end{aligned}
$$

and with the Lorentzian metric $\eta$ in the Minkowski spacetime we have

$$
\left\{\tilde{\gamma}_{c}^{\mu}, \tilde{\gamma}_{c}^{\nu}\right\}=2 \eta^{\mu \nu}, \quad \mu, \nu=t, r, \theta, \phi, \quad \eta^{r r}=\eta^{\theta \theta}=\eta^{\phi \phi}=-1, \quad \eta^{\mu \nu}=0 \quad \text { if } \quad \mu \neq \nu .
$$

Proposition 2.1 In the spherical coordinates the complementary operator for the Dirac operator in the FLRW spacetime is

$$
\begin{aligned}
& \mathscr{D}^{c o}\left(x, t, \partial_{t}, \partial_{x}\right) \\
:= & i t^{-\frac{\ell}{2}} \gamma^{0}\left(t^{i m} \gamma^{U}+t^{-i m} \gamma^{L}\right) \frac{\partial}{\partial t}+i t^{-\frac{3 \ell}{2}}\left(\tilde{\gamma}_{c}^{r} \partial_{r}+\tilde{\gamma}_{c}^{\phi} \frac{1}{r \sin (\theta)} \partial_{\phi}+\tilde{\gamma}_{c}^{\theta} \frac{1}{r} \partial_{\theta}\right)\left(t^{i m} \gamma^{U}+t^{-i m} \gamma^{L}\right),
\end{aligned}
$$

that is,

$$
\begin{aligned}
& \mathscr{D}\left(x, t, \partial_{t}, \partial_{x}\right) \mathscr{D}^{c o}\left(x, t, \partial_{t}, \partial_{x}\right) \\
= & \left(i \gamma^{0} \frac{\partial}{\partial t}+i t^{-\ell}\left(\tilde{\gamma}_{c}^{r} \partial_{r}+\tilde{\gamma}_{c}^{\phi} \frac{1}{r \sin (\theta)} \partial_{\phi}+\tilde{\gamma}_{c}^{\theta} \frac{1}{r} \partial_{\theta}\right)+i \frac{3}{2} \ell t^{-1} \gamma^{0}-m t^{-1} \mathbb{I}_{4}\right) \\
& \times\left(i t^{-\frac{\ell}{2}} \gamma^{0}\left(t^{i m} \gamma^{U}+t^{-i m} \gamma^{L}\right) \frac{\partial}{\partial t}+i t^{-\frac{3 \ell}{2}}\left(\tilde{\gamma}_{c}^{r} \partial_{r}+\tilde{\gamma}_{c}^{\phi} \frac{1}{r \sin (\theta)} \partial_{\phi}+\tilde{\gamma}_{c}^{\theta} \frac{1}{r} \partial_{\theta}\right)\left(t^{i m} \gamma^{U}+t^{-i m} \gamma^{L}\right)\right) \\
= & -t^{-\frac{\ell}{2}}\left(t^{i m} \gamma^{U}+t^{-i m} \gamma^{L}\right)\left\{-\mathbb{I}_{4} \frac{\partial^{2}}{\partial t^{2}}-\left(\ell \mathbb{I}_{4}+2 i m \gamma^{0}\right) \frac{1}{t} \frac{\partial}{\partial t}\right. \\
& \left.+t^{-2 \ell}\left(\frac{\partial^{2}}{\partial r^{2}}+\frac{2}{r} \frac{\partial}{\partial r}+\frac{1}{r^{2}} \frac{\partial^{2}}{\partial \theta^{2}}+\frac{\cot (\theta)}{r^{2}} \frac{\partial}{\partial \theta}+\frac{1}{r^{2} \sin ^{2}(\theta)} \frac{\partial^{2}}{\partial \phi^{2}}\right) \mathbb{I}_{4}\right\}
\end{aligned}
$$

In particular, for the Dirac equation with the source term $F$ we have

$$
i \partial_{t} \Psi+i \frac{1}{a(t)} \gamma^{0}\left(\gamma^{r} \partial_{r}+\gamma^{\phi} \partial_{\phi}+\gamma^{\theta} \partial_{\theta}\right) \Psi+i \frac{3 \dot{a}(t)}{2 a(t)} \Psi-m t^{-1} \gamma^{0} \Psi(x, t)=F(x, t),
$$

where in this Cartesian tetrad gauge the gamma matrices are given by (17),(18),(19). 


\section{Solution of the Cauchy problem}

If we want to solve the problem

$$
\left\{\begin{array}{l}
\mathscr{D}\left(x, t, \partial_{t}, \partial_{x}\right) \Psi(x, t)=F(x, t), \quad t>\varepsilon>0 \\
\Psi(x, \varepsilon)=\Psi_{\varepsilon}(x)
\end{array}\right.
$$

where $\mathscr{D}\left(x, t, \partial_{t}, \partial_{x}\right)$ is given by (14), then it suffices to solve the scalar equations given by the operator

$$
P\left(t, \partial_{t}, \partial_{x} ; m\right):=\partial_{t}^{2}-t^{-2 \ell} \mathcal{A}\left(x, \partial_{x}\right)+t^{-1}(\ell+2 i m) \partial_{t} .
$$

For $\mathcal{A}\left(x, \partial_{x}\right)=\Delta$ the operator $P\left(x, t, \partial_{t}, \partial_{x} ; m\right)$ coincides with the second-order scalar hyperbolic operator (12). Indeed, if we look for the solution in the form

$$
\Psi(x, t)=\mathscr{D}^{c o}\left(x, t, \partial_{t}, \partial_{x}\right) \Phi(x, t),
$$

then (20) implies

$$
\left\{\begin{array}{cc}
-\left(\begin{array}{cc}
t^{-a} \mathbb{I}_{2} P\left(x, t, \partial_{t}, \partial_{x} ; m\right) & \mathbb{O}_{2} \\
\mathbb{O}_{2} & t^{-\omega} \mathbb{I}_{2} P\left(x, t, \partial_{t}, \partial_{x} ;-m\right)
\end{array}\right)\left(\begin{array}{l}
\Phi_{U}(x, t) \\
\Phi_{L}(x, t)
\end{array}\right)=\left(\begin{array}{c}
F_{U}(x, t) \\
F_{L}(x, t)
\end{array}\right), \\
\mathscr{D}^{c o}\left(x, \varepsilon, \partial_{t}, \partial_{x}\right) \Phi(x, \varepsilon)=\Psi_{\varepsilon}(x),
\end{array}\right.
$$

where

$$
F(x, t)=\left(\begin{array}{c}
F_{U}(x, t) \\
F_{L}(x, t)
\end{array}\right), \quad \Phi(x, t)=\left(\begin{array}{c}
\Phi_{U}(x, t) \\
\Phi_{L}(x, t)
\end{array}\right)
$$

Hence,

$$
\left\{\begin{array}{l}
P\left(x, t, \partial_{t}, \partial_{x} ; m\right) \Phi_{U}(x, t)=-t^{a} F_{U}(x, t), \quad t \geq \varepsilon>0 \\
P\left(x, t, \partial_{t}, \partial_{x} ;-m\right) \Phi_{L}(x, t)=-t^{\omega} F_{L}(x, t), \quad t \geq \varepsilon>0 \\
\left.\mathscr{D}^{c o}\left(x, \varepsilon, \partial_{t}, \partial_{x}\right) \Phi(x, t)\right|_{t=\varepsilon}=\Psi_{\varepsilon}(x)
\end{array}\right.
$$

Consider the case of $\Psi_{\varepsilon}(x)=0$, then the functions $\Phi_{U}(x, t)$ and $\Phi_{L}(x, t)$ are given by Theorem 4.1 with vanishing initial data. For the case of $F(x, t)=0$ we choose the zero for the first initial data for the second order equations, that is, $\Phi(x, \varepsilon)=0$. Then

$$
\begin{aligned}
\left.\mathscr{D}^{c o}\left(x, t, \partial_{t}, \partial_{x}\right) \Phi(x, t)\right|_{t=\varepsilon} & =\left.i \varepsilon^{-i m-\frac{\ell}{2}} \gamma^{0}\left(\varepsilon^{2 i m} \gamma^{U}+\gamma^{L}\right) \frac{\partial}{\partial t} \Phi(x, t)\right|_{t=\varepsilon} \\
& =\left.i \varepsilon^{-i m-\frac{\ell}{2}}\left(\begin{array}{cc}
\varepsilon^{2 i m} \mathbb{I}_{2} & \mathbb{O}_{2} \\
\mathbb{O}_{2} & -\mathbb{I}_{2}
\end{array}\right) \frac{\partial}{\partial t}\left(\begin{array}{c}
\Phi_{U}(x, t) \\
\Phi_{L}(x, t)
\end{array}\right)\right|_{t=\varepsilon} .
\end{aligned}
$$

Thus, we have obtained initial conditions for the solutions of the Klein-Gordon equations:

$$
\left\{\begin{array}{l}
P\left(x, t, \partial_{t}, \partial_{x} ; m\right) \Phi_{U}(x, t)=0, \quad t \geq \varepsilon>0 \\
P\left(x, t, \partial_{t}, \partial_{x} ;-m\right) \Phi_{L}(x, t)=0, \quad t \geq \varepsilon>0, \\
\left(\begin{array}{c}
\Phi_{U}(x, \varepsilon) \\
\Phi_{L}(x, \varepsilon)
\end{array}\right)=\left(\begin{array}{c}
0 \\
0
\end{array}\right), \\
\left.\frac{\partial}{\partial t}\left(\begin{array}{c}
\Phi_{U}(x, t) \\
\Phi_{L}(x, t)
\end{array}\right)\right|_{t=\varepsilon}=\left(\begin{array}{c}
-i \varepsilon^{\frac{\ell}{2}-i m} \Psi_{\varepsilon U}(x, \varepsilon) \\
i \varepsilon^{\frac{\ell}{2}+i m} \Psi_{\varepsilon L}(x, \varepsilon)
\end{array}\right) .
\end{array}\right.
$$

The solution to the last problem is given by Theorem 4.1. Let $A\left(x, \partial_{x}\right)=\sum_{|\alpha| \leq d} a_{\alpha}(x) \partial_{x}^{\alpha}$ be a differential operator with the smooth coefficients $a_{\alpha}(x)$. According to Theorem 4.1 if the function $v=v_{f}(x, r ; b)$ solves the problem

$$
\left\{\begin{array}{l}
v_{r r}(x, r ; b)-\mathcal{A}\left(x, \partial_{x}\right) v(x, r ; b)=0 \\
v(x, 0 ; b)=f(x, b), \quad v_{\tau}(x, 0 ; b)=0
\end{array}\right.
$$


while the function $v=v_{\varphi}(x, r)$ solves the problem

$$
\left\{\begin{array}{l}
v_{r r}(x, r)-\mathcal{A}\left(x, \partial_{x}\right) v(x, r)=0 \\
v(x, 0)=\varphi(x), \quad v(x, 0)=0
\end{array}\right.
$$

then the function $u=u(x, t)$ defined by

$$
\begin{aligned}
u(x, t)= & 2 \int_{\varepsilon}^{t} d b \int_{0}^{\phi(t)-\phi(b)} E(r, t ; b ; m) v_{f}(x, r ; b) d r \\
& +\int_{0}^{\phi(t)-\phi(\varepsilon)} \varepsilon(1-\ell)^{-1} K_{1}(r, t ; m ; \varepsilon) v_{\varphi_{1}}(x, r) d r
\end{aligned}
$$

where the kernels $E(r, t ; b ; m)$ and $K_{1}(r, t ; m ; \varepsilon)$ are defined by $(8)$ and $(9)$, respectively, is a solution to the Cauchy problem

$$
\left\{\begin{array}{l}
u_{t t}-t^{-2 \ell} \mathcal{A}\left(x, \partial_{x}\right) u+t^{-1}(\ell+2 i m) u_{t}=f(x, t), \\
u(x, \varepsilon)=0, \quad u_{t}(x, \varepsilon)=\varphi_{1}(x) .
\end{array}\right.
$$

Here and henceforth in the notations $v=v_{f}(x, r ; b)$ and $v=v_{\varphi}(x, r)$ the subscripts $f$ and $\varphi$ do not denote a partial derivative.

Thus, for the 2-spinor solution $\Phi_{U}=\Phi_{U}(x, t)$ of the problem

$$
\left\{\begin{array}{l}
P\left(x, t, \partial_{t}, \partial_{x} ; m\right) \Phi_{U}(x, t)=-t^{a} F_{U}(x, t), \quad t \geq \varepsilon>0, \\
\Phi_{U}(x, \varepsilon)=0, \quad\left(\partial_{t} \Phi_{U}\right)(x, \varepsilon)=-i \varepsilon^{\frac{\ell}{2}-i m} \Psi_{\varepsilon U}(x),
\end{array}\right.
$$

we obtain

$$
\begin{aligned}
\Phi_{U}(x, t)= & -2 \int_{\varepsilon}^{t} b^{\frac{\ell}{2}-i m} d b \int_{0}^{\phi(t)-\phi(b)} E(r, t ; b ; m) \int_{\mathbb{R}^{3}} \mathcal{E}^{w}(x-y, r) F_{U}(y, b) d y d r \\
& -i \varepsilon^{\frac{\ell}{2}-i m} \int_{0}^{\phi(t)-\phi(\varepsilon)} \varepsilon(1-\ell)^{-1} K_{1}(r, t ; m ; \varepsilon) \int_{\mathbb{R}^{3}} \mathcal{E}^{w}(x-y, r) \Psi_{\varepsilon}(y) d y d r, \quad t>\varepsilon>0 .
\end{aligned}
$$

Similarly, for the 2-spinor function $\Phi_{L}=\Phi_{L}(x, t)$ we obtain

$$
\begin{aligned}
\Phi_{L}(x, t)= & -2 \int_{\varepsilon}^{t} b^{\frac{\ell}{2}+i m} d b \int_{0}^{\phi(t)-\phi(b)} E(r, t ; b ;-m) \int_{\mathbb{R}^{3}} \mathcal{E}^{w}(x-y, r) F_{L}(y, b) d y d r \\
& +i \varepsilon^{\frac{\ell}{2}+i m} \int_{0}^{\phi(t)-\phi(\varepsilon)} \varepsilon(1-\ell)^{-1} K_{1}(r, t ;-m ; \varepsilon) \int_{\mathbb{R}^{3}} \mathcal{E}^{w}(x-y, r) \Psi_{\varepsilon L}(y) d y d r, \quad t>\varepsilon>0 .
\end{aligned}
$$

Hence, according to (21), the solution to the Cauchy problem

$$
\left\{\begin{array}{l}
\mathscr{D}\left(x, t, \partial_{t}, \partial_{x}\right) \Psi(x, t)=F(x, t), \quad t>\varepsilon>0 \\
\Psi(x, \varepsilon)=\Psi_{\varepsilon}(x)
\end{array}\right.
$$

for $t>\varepsilon>0$ is given as follows

$$
\begin{aligned}
\Psi(x, t)= & \left\{i t^{-\frac{\ell}{2}} \gamma^{0}\left(t^{i m} \gamma^{U}+t^{-i m} \gamma^{L}\right) \frac{\partial}{\partial t}+i t^{-\frac{3 \ell}{2}} \sum_{l=1}^{3} \gamma^{l}\left(t^{i m} \gamma^{U}+t^{-i m} \gamma^{L}\right) \frac{\partial}{\partial x_{l}}\right\} \\
& \times\left\{\left[-2 \int_{\varepsilon}^{t} b^{\frac{\ell}{2}-i m} d b \int_{0}^{\phi(t)-\phi(b)} E(r, t ; b ; m) \int_{\mathbb{R}^{3}} \mathcal{E}^{w}(x-y, r) F_{U}(y, b) d y d r\right.\right. \\
& \left.-i \varepsilon^{\frac{\ell}{2}-i m} \int_{0}^{\phi(t)-\phi(\varepsilon)} \varepsilon(1-\ell)^{-1} K_{1}(r, t ; m ; \varepsilon) \int_{\mathbb{R}^{3}} \mathcal{E}^{w}(x-y, r) \Psi_{\varepsilon}(y) d y d r\right] \gamma^{U} \\
& +\left[-2 \int_{\varepsilon}^{t} b^{\frac{\ell}{2}+i m} d b \int_{0}^{\phi(t)-\phi(b)} E(r, t ; b ;-m) \int_{\mathbb{R}^{3}} \mathcal{E}^{w}(x-y, r) F_{L}(y, b) d y d r\right. \\
& \left.\left.+i \varepsilon^{\frac{\ell}{2}+i m} \int_{0}^{\phi(t)-\phi(\varepsilon)} \varepsilon(1-\ell)^{-1} K_{1}(r, t ;-m ; \varepsilon) \int_{\mathbb{R}^{3}} \mathcal{E}^{w}(x-y, r) \Psi_{\varepsilon L}(y) d y d r\right] \gamma^{L}\right\}
\end{aligned}
$$


If we introduce the operator $\mathcal{G}\left(x, t, D_{x} ; m\right)$ by

$$
\begin{aligned}
& \mathcal{G}\left(x, t, D_{x} ; m\right)[f](x . t) \\
= & -2 \int_{\varepsilon}^{t} b^{\frac{\ell}{2}-i m} d b \int_{0}^{\phi(t)-\phi(b)} E(r, t ; b ; m) \int_{\mathbb{R}^{3}} \mathcal{E}^{w}(x-y, r) f(y, b) d y d r, \quad f \in C_{0}^{\infty}\left(\mathbb{R}^{n+1}\right),
\end{aligned}
$$

and the operator $\mathcal{K}_{1}\left(x, t, D_{x} ; m ; \varepsilon\right)$ as follows:

$$
\begin{aligned}
& \mathcal{K}_{1}\left(x, t, D_{x} ; m ; \varepsilon\right)[\varphi](x . t) \\
= & -i \varepsilon^{\frac{\ell}{2}-i m} \int_{0}^{\phi(t)-\phi(\varepsilon)} \varepsilon(1-\ell)^{-1} K_{1}(r, t ; m ; \varepsilon) \int_{\mathbb{R}^{3}} \mathcal{E}^{w}(x-y, r) \varphi(y) d y d r, \quad \varphi \in C_{0}^{\infty}\left(\mathbb{R}^{n}\right),
\end{aligned}
$$

then the formula for the solution is

$$
\begin{aligned}
& \Psi(x, t)=\left\{i t^{-\frac{\ell}{2}} \gamma^{0}\left(t^{i m} \gamma^{U}+t^{-i m} \gamma^{L}\right) \frac{\partial}{\partial t}+i t^{-\frac{3 \ell}{2}} \sum_{l=1}^{3} \gamma^{l}\left(t^{i m} \gamma^{U}+t^{-i m} \gamma^{L}\right) \frac{\partial}{\partial x_{l}}\right\} \\
& \times\left\{\left[\mathcal{G}\left(x, t, D_{x} ; m\right)\left[F_{U}\right](x, t)+\mathcal{K}_{1}\left(x, t, D_{x} ; m ; \varepsilon\right)\left[\Psi_{\varepsilon U}\right](x, t)\right] \gamma^{U}\right. \\
& \left.+\left[\mathcal{G}\left(x, t, D_{x} ;-m\right)\left[F_{U}\right](x, t)-\mathcal{K}_{1}\left(x, t, D_{x} ;-m ; \varepsilon\right)\left[\Psi_{\varepsilon L}\right](x, t)\right] \gamma^{L}\right\} \\
& =\left\{i t^{-\frac{\ell}{2}} \gamma^{0}\left(t^{i m} \gamma^{U}+t^{-i m} \gamma^{L}\right) \frac{\partial}{\partial t}+i t^{-\frac{3 \ell}{2}} \sum_{l=1}^{3} \gamma^{l}\left(t^{i m} \gamma^{U}+t^{-i m} \gamma^{L}\right) \frac{\partial}{\partial x_{l}}\right\} \\
& \times\left\{\begin{array}{cc}
\mathcal{G}\left(x, t, D_{x} ; m\right) \mathbb{I}_{2} & \mathbb{O}_{2} \\
\mathbb{O}_{2} & \mathcal{G}\left(x, t, D_{x} ;-m\right) \mathbb{I}_{2}
\end{array}\right)[F](x, t) \\
& \left.+\gamma^{0}\left(\begin{array}{cc}
\mathcal{K}_{1}\left(x, t, D_{x} ; m ; \varepsilon\right) \mathbb{I}_{2} & \mathbb{O}_{2} \\
\mathbb{O}_{2} & \mathcal{K}_{1}\left(x, t, D_{x} ;-m ; \varepsilon\right) \mathbb{I}_{2}
\end{array}\right)\left[\Psi_{\varepsilon}\right](x, t)\right\}, \quad t>\varepsilon>0 .
\end{aligned}
$$

Thus, we have proved main Theorem 1.3.

\section{Proof of Theorems 1.1, 1.2}

Theorems 1.1,1.2 follow immediately from Theorem 1.3, since the Cauchy problem for the hyperbolic operator $\mathscr{D}\left(t, \partial_{t}, \partial_{x}\right)$ is well posed in the space of distributions defined in $\mathbb{R}^{3} \times(0, \infty)$.

In terms of $(7)$, the retarded fundamental solution (propagator) $\mathcal{E}_{+}\left(x, t ; x_{0}, t_{0} ; m\right)\left(=\mathcal{E}_{+}\left(x-x_{0}, t ; 0, t_{0} ; m\right)\right)$ with support in the forward cone $D_{+}\left(x_{0}, t_{0}\right), x_{0} \in \mathbb{R}^{n}, t_{0} \in \mathbb{R}_{+}, \quad \operatorname{supp} \mathcal{E}_{+} \subseteq D_{+}\left(x_{0}, t_{0}\right)$, is given by

$$
\begin{aligned}
\mathcal{E}_{+}\left(x, t ; x_{0}, t_{0} ; m\right)= & \mathscr{D}^{c o}\left(x, t, \partial_{t}, \partial_{x}\right)\left(\begin{array}{cc}
\mathcal{G}\left(x, t, D_{x} ; m\right) \mathbb{I}_{2} & \mathbb{O}_{2} \\
\mathbb{O}_{2} & \mathcal{G}\left(x, t, D_{x} ;-m\right) \mathbb{I}_{2}
\end{array}\right)\left[\delta_{x_{0}} \delta_{t_{0}}\right](x, t) \\
= & -2 t_{0}^{\frac{\ell}{2}-i m} \mathscr{D}^{c o}\left(x, t, \partial_{t}, \partial_{x}\right) \\
& \times \int_{0}^{\phi(t)-\phi\left(t_{0}\right)}\left(\begin{array}{cc}
E\left(r, t ; t_{0} ; m\right) \mathbb{I}_{2} & \mathbb{O}_{2} \\
\mathbb{O}_{2} & E\left(r, t ; t_{0} ;-m\right) \mathbb{I}_{2}
\end{array}\right) \mathcal{E}^{w}\left(x-x_{0}, r\right) d r .
\end{aligned}
$$

We set $0<\varepsilon<t_{0}$ in the definition of the operator $\mathcal{G}\left(x, t, D_{x} ; m\right)$. Hence, for the upper 2-spinor we have

$$
\mathcal{G}\left(x, t, D_{x} ; m\right)\left[\delta_{x_{0}} \delta_{t_{0}}\right](x, t)=-2 t_{0}^{\frac{\ell}{2}-i m} \int_{0}^{\phi(t)-\phi\left(t_{0}\right)} E\left(r, t ; t_{0} ; m\right) \mathcal{E}^{w}\left(x-x_{0}, r\right) d r, \quad t>\varepsilon>0 .
$$

Similarly, for the lower 2-spinor we obtain

$$
\mathcal{G}\left(x, t, D_{x} ;-m\right)\left[\delta_{x_{0}} \delta_{t_{0}}\right](x, t)=-2 t_{0}^{\frac{\ell}{2}+i m} \int_{0}^{\phi(t)-\phi\left(t_{0}\right)} E\left(r, t ; t_{0} ;-m\right) \mathcal{E}^{w}\left(x-x_{0}, r\right) d r, \quad t>\varepsilon>0
$$

Thus, we have proved Theorem 1.1. 


\section{Solution to non-Fuchsian type hyperbolic partial differential equations}

\subsection{Change to the proper time}

In this and remaining sections we assume that $x \in \mathbb{R}^{n}$ with $n \geq 1$. Hence, we have to solve the non-Fuchsian type hyperbolic partial differential equation

$$
u_{t t}-t^{-2 \ell} \Delta u+t^{-1}(\ell+2 i m) u_{t}=f,
$$

where $\Delta$ is Laplacian in $\mathbb{R}^{n}$. After change to comoving frame with the inverse function $\phi^{-1}(\varepsilon)$ if $\ell \neq 1$,

$$
\phi(t)=\frac{1}{1-\ell} t^{1-\ell}, \quad \phi^{-1}(\varepsilon)=|1-\ell|^{\frac{1}{1-\ell}} \varepsilon^{\frac{1}{1-\ell}}, \quad \ell \neq 1 .
$$

We consider the case of $\ell<1$, since the corresponding modifications for the case of $\ell>1$ are evident. Since we want to solve problem with the initial data prescribed on $t=\varepsilon$, we introduce a new variable $s$,

$$
s=\frac{\phi(t)}{\phi(\varepsilon)}=\left(\frac{t}{\varepsilon}\right)^{1-\ell}, \quad t=(1-\ell)^{-\frac{1}{1-\ell}} \varepsilon \phi^{-1}(s)=\varepsilon s^{\frac{1}{1-\ell}},
$$

and obtain for the derivatives

$$
\begin{aligned}
& \frac{d}{d t}=\frac{\phi^{\prime}(t)}{\phi(\varepsilon)} \frac{d}{d s}=\frac{1}{\phi(\varepsilon)} t^{-\ell} \frac{d}{d s}, \quad \frac{\phi^{\prime}(t)}{\phi(\varepsilon)}=(1-\ell)^{-\frac{\ell}{1-\ell}} \frac{1}{\phi(\varepsilon)}(\phi(t))^{-\frac{\ell}{1-\ell}}, \\
& \frac{d^{2}}{d t^{2}}=\left(\frac{\phi^{\prime}(t)}{\phi(\varepsilon)}\right)^{2} \frac{d^{2}}{d s^{2}}+\frac{\phi^{\prime \prime}(t)}{\phi(\varepsilon)} \frac{d}{d s}=\frac{1}{\phi^{2}(\varepsilon)} t^{-2 \ell} \frac{d^{2}}{d s^{2}}-\frac{\ell}{\phi(\varepsilon)} t^{-\ell-1} \frac{d}{d s} .
\end{aligned}
$$

The time $t=\varepsilon$ corresponds to $s=1$. Then the equation (22) for the function $u=u(x, s)$ reads

$$
u_{s s}(x, s)-\phi^{2}(\varepsilon) \Delta u(x, s)+\frac{2 i m}{(1-\ell) s} u_{s}(x, s)=\varepsilon^{2 \ell} \phi^{2}(\varepsilon) s^{\frac{2 \ell}{1-\ell}} f\left(x, \varepsilon s^{1 /(1-\ell)}\right) .
$$

Thus, we arrive at the generalized Fuchsian type partial differential equation (or generalized Euler-PoissonDarboux equation)

$$
\widetilde{u}_{\tau \tau}(x, \tau)-\widetilde{A}\left(x, \partial_{x}\right) \widetilde{u}(x, \tau)+\frac{2 i \widetilde{m}}{\tau+1} \widetilde{u}_{\tau}(x, \tau)=\widetilde{f}(x, \tau)
$$

that will be discussed in the next sections. Here $\widetilde{m}:=\frac{m}{1-\ell}, \tau=s-1$, and for the case of (22) we have $A\left(x, \partial_{x}\right)=\Delta$, while in general,

$$
\begin{aligned}
& \widetilde{A}\left(x, \partial_{x}\right)=\phi^{2}(\varepsilon) A\left(x, \partial_{x}\right), \\
& \widetilde{f}(x, \tau)=\left(\frac{\phi(\varepsilon)}{\phi^{\prime}(t)}\right)^{2} f\left(x, \varepsilon(\tau+1)^{1 /(1-\ell)}\right), \\
& \widetilde{f}(x, \tau)=(1-\ell)^{\frac{2 \ell}{1-\ell}} \phi^{\frac{2}{1-\ell}}(\varepsilon)(\tau+1)^{\frac{2 \ell}{1-\ell}} f\left(x, \varepsilon(\tau+1)^{1 /(1-\ell)}\right), \\
& t=\varepsilon(\tau+1)^{1 /(1-\ell)}, \quad \tau=\frac{\phi(t)-\phi(\varepsilon)}{\phi(\varepsilon)},
\end{aligned}
$$

where $A\left(x, \partial_{x}\right)$ is a pseudo-differential operator of order $d$. For the Dirac operator written in Cartesian coordinates $A\left(x, \partial_{x}\right)=\Delta$. On the other hand, the Dirac operator written in other orthogonal coordinate systems is rather complicated partial differential operator with the variable coefficients depending not only on time variable (16) (see, also, [24, 33]). Having this in mind, in what follows, we discuss more general case (23) of the operator $A\left(x, \partial_{x}\right)$ that can be an operator of order $d$ higher than two. 


\subsection{Solution in the original time}

To write a solution to the Cauchy problem for the operator $P\left(t, \partial_{t}, \partial_{t} ; m\right)$ we need one more kernel function that is defined by means of $K_{0}(r, \tau ; m)$ of subsection 5.1 as follows

$$
\begin{aligned}
& K_{0}(r, t ; m ; \varepsilon):=K_{0}\left(\frac{r}{\phi(\varepsilon)}, \frac{\phi(t)-\phi(\varepsilon)}{\phi(\varepsilon)} ; \frac{m}{1-\ell}\right) \\
= & -2^{2 i \frac{m}{1-\ell}} \frac{m}{1-\ell} \phi^{\frac{2 i m}{1-\ell}}(\varepsilon)\left((\phi(t)+\phi(\varepsilon))^{2}-r^{2}\right)^{-i \frac{m}{1-\ell}} \\
& \times\left\{\frac{2 i\left(r^{2}-\phi(t)(\phi(t)-\phi(\varepsilon))\right)}{r^{2}-(\phi(t)-\phi(\varepsilon))^{2}} F\left(i \frac{m}{1-\ell}, i \frac{m}{1-\ell} ; 1 ; \frac{(\phi(t)-\phi(\varepsilon))^{2}-r^{2}}{(\phi(t)+\phi(\varepsilon))^{2}-r^{2}}\right)\right. \\
& -\frac{4 i \phi(t) \phi(\varepsilon)\left(\phi^{2}(t)-\phi^{2}(\varepsilon)-r^{2}\right)}{\left((\phi(t)-\phi(\varepsilon))^{2}-r^{2}\right)\left((\phi(t)+\phi(\varepsilon))^{2}-r^{2}\right)} \\
& \left.\times F\left(i \frac{m}{1-\ell}+1, i \frac{m}{1-\ell} ; 1 ; \frac{(\phi(t)-\phi(\varepsilon))^{2}-r^{2}}{(\phi(t)+\phi(\varepsilon))^{2}-r^{2}}\right)\right\} .
\end{aligned}
$$

Since the following results cover a wide class of pseudo-differential operators $A\left(x, \partial_{x}\right)$ and even abstract linear operators, we will not specify Sobolev spaces or distributions, and simply assume that these functions possess continuous partial derivatives in time variable up to second order.

Theorem 4.1 Assume that the function $v=v_{f}(x, r ; b) \in C_{x, r, b}^{d, 2,0}$ solves the problem

$$
\left\{\begin{array}{l}
v_{r r}(x, r ; b)-A\left(x, \partial_{x}\right) v(x, r ; b)=0 \\
v(x, 0 ; b)=f(x, b), \quad v_{r}(x, 0 ; b)=0
\end{array}\right.
$$

while the function $v=v_{\varphi}(x, r) \in C_{x, r}^{d, 2}$ solves the problem

$$
\left\{\begin{array}{l}
v_{r r}(x, r)-A\left(x, \partial_{x}\right) v(x, r)=0 \\
v(x, 0)=\varphi(x), \quad v_{r}(x, 0)=0
\end{array}\right.
$$

Then the function $u=u(x, t)$ defined by

$$
\begin{aligned}
& u(x, t) \\
= & 2 \int_{\varepsilon}^{t} d b \int_{0}^{\phi(t)-\phi(b)} E(r, t ; b ; m) v_{f}(x, r ; b) d r+\int_{0}^{\phi(t)-\phi(\varepsilon)} \varepsilon(1-\ell)^{-1} K_{1}(r, t ; m ; \varepsilon) v_{\varphi_{1}}(x, r) d r \\
& +\left(\frac{\phi(t)}{\phi(\varepsilon)}\right)^{-i \frac{m}{1-\ell}} v_{\varphi_{0}}(x, \phi(t)-\phi(\varepsilon)) \\
& +\frac{1}{\phi(\varepsilon)} \int_{0}^{\phi(t)-\phi(\varepsilon)}\left[K_{0}(r, t ; m ; \varepsilon)+2 i m K_{1}(r, t ; m ; \varepsilon)\right] v_{\varphi_{0}}(x, r) d r,
\end{aligned}
$$

where the kernels $E(r, t ; b ; m), K_{1}(r, t ; m ; \varepsilon)$, and $K_{0}(r, t ; m ; \varepsilon)$ are defined by (8), (9), and (27), respectively, is a solution to the Cauchy problem

$$
\left\{\begin{array}{l}
u_{t t}-t^{-2 \ell} A\left(x, \partial_{x}\right) u+t^{-1}(\ell+2 i m) u_{t}=f(x, t), \quad t>\varepsilon \\
u(x, \varepsilon)=\varphi_{0}(x), \quad u_{t}(x, \varepsilon)=\varphi_{1}(x)
\end{array}\right.
$$

In order to prove this theorem we consider separately three cases, which correspond to three functions $f(x, t), \varphi_{1}(x)$, and $\varphi_{0}(x)$.

\subsection{Proof of Theorem 4.1 with $f$. Case of $\varphi_{0}=\varphi_{1}=0$}

According to Theorem 5.3 the function

$$
\widetilde{u}(x, \tau)=\int_{0}^{\tau} d b \int_{0}^{\tau-b} \widetilde{E}(r, \tau ; b ; \widetilde{m}) \widetilde{v}_{\widetilde{f}}(x, r ; b) d r,
$$


where

$$
\widetilde{E}(r, \tau ; b ; \widetilde{m}):=2^{2 i \widetilde{m}}(1+b)^{2 i \widetilde{m}}\left((\tau+b+2)^{2}-r^{2}\right)^{-i \widetilde{m}} F\left(i \widetilde{m}, i \widetilde{m} ; 1 ; \frac{(\tau-b)^{2}-r^{2}}{(\tau+b+2)^{2}-r^{2}}\right)
$$

and the function $\widetilde{v}=\widetilde{v}_{\widetilde{f}}(x, r ; b) \in C_{x, r, b}^{d, 2,0}$ is defined by

$$
\left\{\begin{array}{l}
\widetilde{v}_{r r}(x, r ; b)-\widetilde{A}\left(x, \partial_{x}\right) \widetilde{v}(x, r ; b)=0, \\
\widetilde{v}(x, 0 ; b)=\widetilde{f}(x, b), \quad \widetilde{v}_{r}(x, 0 ; b)=0,
\end{array}\right.
$$

solves the Cauchy problem

$$
\left\{\begin{array}{l}
\widetilde{u}_{\tau \tau}(x, \tau)-\widetilde{A}\left(x, \partial_{x}\right) \widetilde{u}(x, \tau)+\frac{2 i \widetilde{m}}{\tau+1} \widetilde{u}_{\tau}(x, \tau)=\widetilde{f}(x, \tau) \\
\widetilde{u}(x, 0)=0, \quad \widetilde{u}_{\tau}(x, 0)=0
\end{array}\right.
$$

Lemma 4.2 Assume that the function $z=z_{f}(x, r ; b) \in C_{x, r, b}^{d, 2,0}$ solves the problem

$$
\left\{\begin{array}{l}
z_{r r}(x, r ; b)-A\left(x, \partial_{x}\right) z(x, r ; b)=0 \\
z(x, 0 ; b)=f(x, b), \quad z_{r}(x, 0 ; b)=0
\end{array}\right.
$$

then

$$
\widetilde{v}_{\widetilde{f}}(x, r ; b)=(1-\ell)^{\frac{2 \ell}{1-\ell}} \phi^{\frac{2}{1-\ell}}(\varepsilon)(\tau+1)^{\frac{2 \ell}{1-\ell}} z_{f}\left(x, \phi(\varepsilon) r ; \varepsilon(b+1)^{1 /(1-\ell)}\right)
$$

solves the problem

$$
\left\{\begin{array}{l}
\widetilde{v}_{r r}(x, r ; b)-\widetilde{A}\left(x, \partial_{x}\right) \widetilde{v}(x, r ; b)=0, \\
\widetilde{v}(x, 0 ; b)=\widetilde{f}(x, b), \quad \widetilde{v}_{r}(x, 0 ; b)=0,
\end{array}\right.
$$

where $\widetilde{A}\left(x, \partial_{x}\right)=\phi^{2}(\varepsilon) A\left(x, \partial_{x}\right)$ and

$$
\tilde{f}(x, \tau)=(1-\ell)^{\frac{2 \ell}{1-\ell}} \phi^{\frac{2}{1-\ell}}(\varepsilon)(\tau+1)^{\frac{2 \ell}{1-\ell}} f\left(x, \varepsilon(\tau+1)^{1 /(1-\ell)}\right) .
$$

Proof. According to (24) and (25), we obtain

$$
\widetilde{v}_{\widetilde{f}}(x, r ; b)=(1-\ell)^{\frac{2 \ell}{1-\ell}} \phi^{\frac{2}{1-\ell}}(\varepsilon)(b+1)^{\frac{2 \ell}{1-\ell}} v_{f}(x, r ; b),
$$

where the function $v=v_{f}(x, r ; b)$ is defined by

$$
\left\{\begin{array}{l}
v_{r r}(x, r ; b)-\widetilde{A}\left(x, \partial_{x}\right) v(x, r ; b)=0, \\
v(x, 0 ; b)=f\left(x, \varepsilon(b+1)^{1 /(1-\ell)}\right), \quad v_{r}(x, 0 ; b)=0 .
\end{array}\right.
$$

Assume that the function $w=w(x, r ; b) \in C_{x, r, b}^{d, 2,0}$ solves

$$
\left\{\begin{array}{l}
w_{r r}(x, r ; b)-\widetilde{A}\left(x, \partial_{x}\right) w(x, r ; b)=0, \\
w(x, 0 ; b)=f(x, b), \quad w_{r}(x, 0 ; b)=0,
\end{array}\right.
$$

then

$$
w(x, r ; b)=z(x, \phi(\varepsilon) r ; b)
$$

Lemma is proved.

Consider with $\tau=\frac{\phi(t)-\phi(\varepsilon)}{\phi(\varepsilon)}$ the function

$$
\begin{aligned}
u(x, t)= & \int_{0}^{\tau} d b \int_{0}^{\tau-b} \widetilde{E}(r, \tau ; b ; \widetilde{m}) \widetilde{v}_{\tilde{f}}(x, r ; b) d r \\
= & \int_{0}^{\frac{\phi(t)-\phi(\varepsilon)}{\phi(\varepsilon)}} d b \int_{0}^{\frac{\phi(t)-\phi(\varepsilon)}{\phi(\varepsilon)}-b} \widetilde{E}\left(r, \frac{\phi(t)-\phi(\varepsilon)}{\phi(\varepsilon)} ; b ; \widetilde{m}\right) \\
& \times(1-\ell)^{\frac{2 \ell}{1-\ell}} \phi^{\frac{2}{1-\ell}}(\varepsilon)(b+1)^{\frac{2 \ell}{1-\ell}} z_{f}\left(x, \phi(\varepsilon) r ; \varepsilon(b+1)^{1 /(1-\ell)}\right) d r .
\end{aligned}
$$


Using the substitution $y=\phi(\varepsilon) r$, we obtain

$$
\begin{aligned}
u(x, t)= & \frac{1}{\phi(\varepsilon)} \int_{0}^{\frac{\phi(t)-\phi(\varepsilon)}{\phi(\varepsilon)}} d b \int_{0}^{\phi(t)-(b+1) \phi(\varepsilon)} \widetilde{E}\left(\frac{y}{\phi(\varepsilon)}, \frac{\phi(t)-\phi(\varepsilon)}{\phi(\varepsilon)} ; b ; \widetilde{m}\right) \\
& \times(1-\ell)^{\frac{2 \ell}{1-\ell}} \phi^{\frac{2}{1-\ell}}(\varepsilon)(b+1)^{\frac{2 \ell}{1-\ell}} z_{f}\left(x, y ; \varepsilon(b+1)^{1 /(1-\ell)}\right) d y .
\end{aligned}
$$

Introducing $\eta=\varepsilon(b+1)^{1 /(1-\ell)}$ and using the following relations

$$
\begin{aligned}
& b+1=\frac{\phi(\eta)}{\phi(\varepsilon)}, \quad b=\frac{\phi(\eta)-\phi(\varepsilon)}{\phi(\varepsilon)}, \quad d b=\frac{\phi^{\prime}(\eta)}{\phi(\varepsilon)} d \eta, \\
& b=0 \longmapsto \eta=\varepsilon, \quad b=\frac{\phi(t)-\phi(\varepsilon)}{\phi(\varepsilon)} \longmapsto \eta=t,
\end{aligned}
$$

we obtain

$$
\begin{aligned}
u(x, t)=(\phi(\varepsilon))^{-1}(1-\ell)^{\frac{2 \ell}{1-\ell}} \phi^{\frac{2}{1-\ell}}(\varepsilon) \int_{\varepsilon}^{t} d \eta \int_{0}^{\phi(t)-\phi(\eta)} \frac{\phi^{\prime}(\eta)}{\phi(\varepsilon)}\left(\frac{\phi(\eta)}{\phi(\varepsilon)}\right)^{2 \ell /(1-\ell)} \\
\times \widetilde{E}\left(\frac{y}{\phi(\varepsilon)}, \frac{\phi(t)-\phi(\varepsilon)}{\phi(\varepsilon)} ; \frac{\phi(\eta)-\phi(\varepsilon)}{\phi(\varepsilon)} ; \widetilde{m}\right) z_{f}(x, y ; \eta) d y
\end{aligned}
$$

Now we use

$$
\phi(\eta)=\frac{1}{1-\ell} \eta^{1-\ell}, \quad \phi^{\prime}(\eta)=\eta^{-\ell}, \quad \eta=[(1-\ell) \phi(\eta)]^{\frac{1}{1-\ell}}, \quad \phi^{\prime}(\eta)=(1-\ell)^{\frac{-\ell}{1-\ell}} \phi(\eta)^{\frac{-\ell}{1-\ell}}
$$

and derive

$$
\begin{aligned}
u(x, t)= & (\phi(\varepsilon))^{-2}(1-\ell)^{\frac{\ell}{1-\ell}} \phi^{\frac{2}{1-\ell}}(\varepsilon) \int_{\varepsilon}^{t} d \eta \int_{0}^{\phi(t)-\phi(\eta)} \phi(\eta)^{\frac{-\ell}{1-\ell}}\left(\frac{\phi(\eta)}{\phi(\varepsilon)}\right)^{2 \ell /(1-\ell)} \\
& \times \widetilde{E}\left(\frac{y}{\phi(\varepsilon)}, \frac{\phi(t)-\phi(\varepsilon)}{\phi(\varepsilon)} ; \frac{\phi(\eta)-\phi(\varepsilon)}{\phi(\varepsilon)} ; \widetilde{m}\right) z_{f}(x, y ; \eta) d y \\
= & (1-\ell)^{\frac{\ell}{1-\ell}} \int_{\varepsilon}^{t} \phi(\eta)^{\frac{\ell}{1-\ell}} d \eta \int_{0}^{\phi(t)-\phi(\eta)} \\
& \times \widetilde{E}\left(\frac{y}{\phi(\varepsilon)}, \frac{\phi(t)-\phi(\varepsilon)}{\phi(\varepsilon)} ; \frac{\phi(\eta)-\phi(\varepsilon)}{\phi(\varepsilon)} ; \widetilde{m}\right) z_{f}(x, y ; \eta) d y
\end{aligned}
$$

On the other hand, for the function $\widetilde{E}$ we obtain

$$
\begin{aligned}
& \widetilde{E}\left(\frac{y}{\phi(\varepsilon)}, \frac{\phi(t)-\phi(\varepsilon)}{\phi(\varepsilon)} ; \frac{\phi(\eta)-\phi(\varepsilon)}{\phi(\varepsilon)} ; \widetilde{m}\right) \\
= & 2^{2 i \widetilde{m}}(\phi(\eta))^{2 i \widetilde{m}}\left((\phi(t)+\phi(\eta))^{2}-y^{2}\right)^{-i \widetilde{m}} F\left(i \widetilde{m}, i \widetilde{m} ; 1 ; \frac{(\phi(t)-\phi(\eta))^{2}-y^{2}}{(\phi(t)+\phi(\eta))^{2}-y^{2}}\right)
\end{aligned}
$$

and, consequently,

$$
\begin{aligned}
u(x, t)= & 2^{2 i \widetilde{m}}(1-\ell)^{\frac{\ell}{1-\ell}} \int_{\varepsilon}^{t} \phi(\eta)^{\frac{\ell}{1-\ell}+2 i \widetilde{m}} d \eta \int_{0}^{\phi(t)-\phi(\eta)}\left((\phi(t)+\phi(\eta))^{2}-y^{2}\right)^{-i \widetilde{m}} \\
& \times F\left(i \widetilde{m}, i \widetilde{m} ; 1 ; \frac{(\phi(t)-\phi(\eta))^{2}-y^{2}}{(\phi(t)+\phi(\eta))^{2}-y^{2}}\right) z_{f}(x, y ; \eta) d y
\end{aligned}
$$

Hence, according to (8), the function

$$
u(x, t)=2 \int_{\varepsilon}^{t} d \eta \int_{0}^{\phi(t)-\phi(\eta)} E(y, t ; \eta ; m) z_{f}(x, y ; \eta) d y
$$

solves equation (22) in accordance to the integral transform approach of [30,31]. Thus, we have proved the case of $\varphi_{0}=\varphi_{1}=0$ of Theorem 4.1. 


\subsection{Proof of Theorem 4.1 with $\varphi_{1}$. Case of $f=0, \varphi_{0}=0$}

According to Theorem 5.3, the function

$$
\widetilde{u}(x, \tau)=\int_{0}^{\tau} K_{1}(r, \tau ; \widetilde{m}) \widetilde{v}_{\varphi_{1}}(x, r) d r
$$

solves the Cauchy problem

$$
\left\{\begin{array}{l}
\widetilde{u}_{\tau \tau}(x, \tau)-\widetilde{A}\left(x, \partial_{x}\right) \widetilde{u}(x, \tau)+\frac{2 i \widetilde{m}}{\tau+1} \widetilde{u}_{\tau}(x, \tau)=0, \\
\widetilde{u}(x, 0)=0, \quad \widetilde{u}_{\tau}(x, 0)=\varphi_{1}(x)
\end{array}\right.
$$

where $\widetilde{v}=\widetilde{v}_{\varphi}(x, r)$ is a solution to the problem

$$
\left\{\begin{array}{l}
\widetilde{v}_{r r}(x, r)-\widetilde{A}\left(x, \partial_{x}\right) \widetilde{v}(x, r)=0 \\
\widetilde{v}(x, 0)=\varphi(x), \quad \widetilde{v}_{r}(x, 0)=0
\end{array}\right.
$$

Note that $v_{\varphi}(x, r)=\widetilde{v}_{\varphi}\left(x, \phi^{-1}(\varepsilon) r\right)$ solves the equation $v_{r r}(x, r)-A\left(x, \partial_{x}\right) v(x, r)=0$. We set $\tau=\frac{\phi(t)-\phi(\varepsilon)}{\phi(\varepsilon)}$ (26) and consider the function

$$
\begin{aligned}
u(x, t)= & \frac{\varepsilon}{1-\ell} \int_{0}^{\tau} K_{1}(r, \tau ; \widetilde{m}) \widetilde{v}_{\varphi_{1}}(x, r) d r \\
= & \frac{\varepsilon}{1-\ell} \int_{0}^{\frac{\phi(t)-\phi(\varepsilon)}{\phi(\varepsilon)}} K_{1}\left(r, \frac{\phi(t)-\phi(\varepsilon)}{\phi(\varepsilon)} ; \widetilde{m}\right) \widetilde{v}_{\varphi_{1}}(x, r) d r \\
= & \frac{\varepsilon}{1-\ell} \int_{0}^{\frac{\phi(t)-\phi(\varepsilon)}{\phi(\varepsilon)}} K_{1}\left(r, \frac{\phi(t)-\phi(\varepsilon)}{\phi(\varepsilon)} ; \widetilde{m}\right) v_{\varphi_{1}}(x, \phi(\varepsilon) r) d r \\
= & \frac{\varepsilon}{1-\ell} \frac{1}{\phi(\varepsilon)} \int_{0}^{\phi(t)-\phi(\varepsilon)} K_{1}\left(\frac{1}{\phi(\varepsilon)} y, \frac{\phi(t)-\phi(\varepsilon)}{\phi(\varepsilon)} ; \widetilde{m}\right) v_{\varphi_{1}}(x, y) d y \\
= & \frac{\varepsilon}{1-\ell} \frac{1}{\phi(\varepsilon)} \int_{0}^{\phi(t)-\phi(\varepsilon)} 2^{2 i \widetilde{m}} \phi(\varepsilon)^{2 i \widetilde{m}}\left((\phi(t)+\phi(\varepsilon))^{2}-y^{2}\right)^{-i \widetilde{m}} \\
& \times F\left(i \widetilde{m}, i \widetilde{m} ; 1 ; \frac{(\phi(t)-\phi(\varepsilon))^{2}-y^{2}}{(\phi(t)+\phi(\varepsilon))^{2}-y^{2}}\right) v_{\varphi_{1}}(x, y) d y, \quad t \geq \varepsilon>0 .
\end{aligned}
$$

Finally,

$$
\begin{aligned}
u(x, t)= & 2^{2 i \widetilde{m}} \phi(\varepsilon)^{2 i \widetilde{m}-1} \frac{\varepsilon}{1-\ell} \int_{0}^{\phi(t)-\phi(\varepsilon)}\left((\phi(t)+\phi(\varepsilon))^{2}-y^{2}\right)^{-i \widetilde{m}} \\
& \times F\left(i \widetilde{m}, i \widetilde{m} ; 1 ; \frac{(\phi(t)-\phi(\varepsilon))^{2}-y^{2}}{(\phi(t)+\phi(\varepsilon))^{2}-y^{2}}\right) v_{\varphi_{1}}(x, y) d y \\
= & \int_{0}^{\phi(t)-\phi(\varepsilon)} \frac{\varepsilon}{1-\ell} K_{1}(r, t ; m ; \varepsilon) v_{\varphi_{1}}(x, r) d r, \quad t \geq \varepsilon>0
\end{aligned}
$$

where (9) has been used. Thus, we have proved the case of $\varphi_{0}(x)=0$ and $f(x, t)=0$ of Theorem 4.1 .

\subsection{Proof of Theorem 4.1 with $\varphi_{0}$. Case of $f=0, \varphi_{1}=0$}

According to Theorem 5.3, if the function $v=v_{\varphi_{0}}(x, r) \in C_{x, r}^{d, 2}$ solves the Cauchy problem

$$
\left\{\begin{array}{l}
\widetilde{v}_{r r}(x, r)-\widetilde{A}\left(x, \partial_{x}\right) \widetilde{v}(x, r)=0, \\
\widetilde{v}(x, 0)=\varphi_{0}(x), \quad \partial_{r} \widetilde{v}(x, 0)=0,
\end{array}\right.
$$


then the function

$$
\widetilde{u}(x, \tau)=(1+\tau)^{-3 i \widetilde{m}} \widetilde{v}_{\varphi_{0}}(x, \tau)+\int_{0}^{\tau}\left[K_{0}(r, \tau ; \widetilde{m})+2 i \widetilde{m} K_{1}(r, \tau ; \widetilde{m})\right] \widetilde{v}_{\varphi_{0}}(x, r) d r
$$

solves the Cauchy problem

$$
\left\{\begin{array}{l}
\widetilde{u}_{\tau \tau}(x, \tau)-\widetilde{A}\left(x, \partial_{x}\right) \widetilde{u}(x, \tau)+\frac{2 i \widetilde{m}}{\tau+1} \widetilde{u}_{\tau}(x, \tau)=0, \\
\widetilde{u}(x, 0)=\varphi_{0}(x), \quad \widetilde{u}_{\tau}(x, 0)=0 .
\end{array}\right.
$$

Note that $v_{\varphi}(x, r)=\widetilde{v}_{\varphi}\left(x, \phi^{-1}(\varepsilon) r\right)$ solves the equation $v_{r r}(x, r)-A\left(x, \partial_{x}\right) v(x, r)=0$. Set $\tau=\frac{\phi(t)-\phi(\varepsilon)}{\phi(\varepsilon)}$ (26), then the function

$$
\begin{aligned}
& u(x, t) \\
& =(1+\tau)^{-i \widetilde{m}} \widetilde{v}_{\varphi_{0}}(x, \tau)+\int_{0}^{\tau}\left[K_{0}(r, \tau ; \widetilde{m})+2 i \widetilde{m} K_{1}(r, \tau ; \widetilde{m})\right] \widetilde{v}_{\varphi_{0}}(x, r) d r \\
& =\left(\frac{\phi(t)}{\phi(\varepsilon)}\right)^{-i \widetilde{m}} \widetilde{v}_{\varphi_{0}}\left(x, \frac{\phi(t)-\phi(\varepsilon)}{\phi(\varepsilon)}\right) \\
& +\int_{0}^{\frac{\phi(t)-\phi(\varepsilon)}{\phi(\varepsilon)}}\left[K_{0}\left(r, \frac{\phi(t)-\phi(\varepsilon)}{\phi(\varepsilon)} ; \widetilde{m}\right)+2 i \widetilde{m} K_{1}\left(r, \frac{\phi(t)-\phi(\varepsilon)}{\phi(\varepsilon)} ; \widetilde{m}\right)\right] \widetilde{v}_{\varphi_{0}}(x, r) d r \\
& =\left(\frac{\phi(t)}{\phi(\varepsilon)}\right)^{-i \widetilde{m}} v_{\varphi_{0}}(x, \phi(t)-\phi(\varepsilon)) \\
& +\int_{0}^{\frac{\phi(t)-\phi(\varepsilon)}{\phi(\varepsilon)}}\left[K_{0}\left(r, \frac{\phi(t)-\phi(\varepsilon)}{\phi(\varepsilon)} ; \widetilde{m}\right)+2 i \widetilde{m} K_{1}\left(r, \frac{\phi(t)-\phi(\varepsilon)}{\phi(\varepsilon)} ; \widetilde{m}\right)\right] v_{\varphi_{0}}(x, \phi(\varepsilon) r) d r \\
& =\left(\frac{\phi(t)}{\phi(\varepsilon)}\right)^{-i \frac{m}{1-\ell}} v_{\varphi_{0}}(x, \phi(t)-\phi(\varepsilon))+\frac{1}{\phi(\varepsilon)} \int_{0}^{\phi(t)-\phi(\varepsilon)}\left[K_{0}\left(\frac{y}{\phi(\varepsilon)}, \frac{\phi(t)-\phi(\varepsilon)}{\phi(\varepsilon)} ; \frac{m}{1-\ell}\right)\right. \\
& \left.+\frac{2 i m}{1-\ell} K_{1}\left(\frac{y}{\phi(\varepsilon)}, \frac{\phi(t)-\phi(\varepsilon)}{\phi(\varepsilon)} ; \frac{m}{1-\ell}\right)\right] v_{\varphi_{0}}(x, y) d y
\end{aligned}
$$

solves the problem

$$
\left\{\begin{array}{l}
u_{t t}-t^{-2 \ell} A\left(x, \partial_{x}\right) u+t^{-1}(\ell+2 i m) u_{t}=0 \\
u(x, \varepsilon)=\varphi_{0}(x), \quad u_{t}(x, \varepsilon)=0
\end{array}\right.
$$

Thus, we have proved the case of $\varphi_{1}(x)=0$ and $f(x, t)=0$ of Theorem 4.1.

\section{$5 \quad$ Integral transform approach to generalized Euler-Poisson-Darboux equation}

From now on in this section we will omit "tilde" in the notations. The rest of this section is devoted to the following generalized Euler-Poisson-Darboux equation

$$
\partial_{\tau}^{2} u-A\left(x, \partial_{x}\right) u+\frac{2 i m}{\tau+1} \partial_{\tau} u=f,
$$

where $m \in \mathbb{C}$ and $A\left(x, \partial_{x}\right)$ is a pseudo-differential operator with the symbol $A(x, \xi)$ defined for $(x, \xi) \in \Omega \times \mathbb{R}^{n}$. Here $\Omega$ is a domain in $\mathbb{R}^{n}$. For the sake of the previous sections it is enough to set $\Omega=\mathbb{R}^{n}$. For the cases with $\Omega \neq \mathbb{R}^{n}$ one can consult [31].

There is a very extensive literature on Euler-Poisson-Darboux equation, that is equation (28) with $A\left(x, \partial_{x}\right)=\Delta$ (see, e.g., $[4,8,9,10,22,26,28,29,31]$ and bibliography therein). In particular, Wirth [29] used Bessel functions to represent the solution of Euler-Poisson-Darboux equation. In [21] the version of 
the integral transform approach [30, 32] was employed by Palmieri for the derivation of the integral representation formulas for the solution of a linear equation with the coefficients, which are scale-invariant and independent of $x$ variable. Then Palmieri and coauthors [14] applied these formulas to examine the blowup phenomena for the wave equations with different types of nonlinearities in the spacetime with power type expansion.

By the integral transform approach suggested in $[30,31,32,35]$ several results presented in the literature can be extended to the generalized Euler-Poisson-Darboux equation (28). Here we present one of such generalizations.

\subsection{Kernels for Generalized Euler-Poisson-Darboux equation.}

To solve the Cauchy problem for (28) with $m \in \mathbb{C}$ and data on $\tau=0$ we use in this section the following kernel functions

$$
\begin{aligned}
E(r, \tau ; b ; m)= & 2^{2 i m}(1+b)^{2 i m}\left((\tau+b+2)^{2}-r^{2}\right)^{-i m} F\left(i m, i m ; 1 ; \frac{(\tau-b)^{2}-r^{2}}{(\tau+b+2)^{2}-r^{2}}\right) \\
K_{1}(r, \tau ; m)= & E(r, \tau ; 0 ; m)=2^{2 i m}\left((\tau+2)^{2}-r^{2}\right)^{-i m} F\left(i m, i m ; 1 ; \frac{\tau^{2}-r^{2}}{(\tau+2)^{2}-r^{2}}\right), \\
K_{0}(r, \tau ; m)= & \lim _{b \rightarrow 0}\left(-\frac{\partial}{\partial b} E(r, \tau ; b ; m)\right) \\
= & -2^{2 i m} m\left((\tau+2)^{2}-r^{2}\right)^{-i m} \\
& \times\left(\frac{2 i\left(r^{2}-\tau(\tau+1)\right)}{r^{2}-\tau^{2}} F\left(i m, i m ; 1 ; \frac{\tau^{2}-r^{2}}{(\tau+2)^{2}-r^{2}}\right)\right. \\
& \left.-\frac{4 i(\tau+1)\left(\tau(\tau+2)-r^{2}\right)}{\left(\tau^{2}-r^{2}\right)\left((\tau+2)^{2}-r^{2}\right)} F\left(i m+1, i m ; 1 ; \frac{\tau^{2}-r^{2}}{(\tau+2)^{2}-r^{2}}\right)\right) .
\end{aligned}
$$

The statements of the next theorem are the consequences of Theorem 2.12 [35], Propositions 2.14, 2.15 [35].

Theorem 5.1 The functions $E, K_{0}$, and $K_{1}$, solve the Euler-Poisson-Darboux equation, that is,

$$
\begin{aligned}
& E_{\tau \tau}(r, \tau ; b ; m)-E_{r r}(r, \tau ; b ; m)+\frac{2 i m}{\tau+1} E_{\tau}(r, \tau ; b ; m)=0, \\
& K_{0 \tau \tau}(r, \tau ; m)-K_{0 r r}(r, \tau ; m)+\frac{2 i m}{\tau+1} K_{0 \tau}(r, \tau ; m)=0, \\
& K_{1 \tau \tau}(r, \tau ; m)-K_{1 r r}(r, \tau ; m)+\frac{2 i m}{\tau+1} K_{1 \tau}(r, \tau ; m)=0,
\end{aligned}
$$

respectively.

Proof. We prove the statement for the function $E$ only, since for two remaining functions the statements follow from the first one. According to Theorem 2.12 [35], the function

$$
W(r, \tau ; b ; M):=4^{-M}(b \tau)^{-M}\left((b+\tau)^{2}-r^{2}\right)^{M-\frac{1}{2}} F\left(\frac{1}{2}-M, \frac{1}{2}-M ; 1 ; \frac{(b-\tau)^{2}-r^{2}}{(b+\tau)^{2}-r^{2}}\right)
$$

solves the following linear partial differential equation with parameters $b$ and $M$ :

$$
W_{\tau \tau}-W_{r r}+\frac{1}{\tau} W_{\tau}-\frac{1}{\tau^{2}} M^{2} W=0 .
$$

Furthermore, for the function $W=\tau^{-M} V$ we obtain

$$
V_{\tau \tau}-V_{r r}+\frac{1-2 M}{\tau} V_{\tau}=0
$$


Hence with $1-2 M=2 i m$

$$
\tau^{-i m+\frac{1}{2}} W\left(r, \tau ; b ; i m-\frac{1}{2}\right)=V\left(r, \tau ; b ; i m-\frac{1}{2}\right)
$$

and the function

$$
V\left(r, \tau ; b ; i m-\frac{1}{2}\right)=2^{2 i m-1}(b)^{-\left(\frac{1}{2}-i m\right)}\left((b+\tau)^{2}-r^{2}\right)^{-i m} F\left(i m, i m ; 1 ; \frac{(b-\tau)^{2}-r^{2}}{(b+\tau)^{2}-r^{2}}\right)
$$

solves the equation

$$
V_{\tau \tau}-V_{r r}+\frac{2 i m}{\tau} V_{\tau}=0 .
$$

It remains to shift $\tau \longrightarrow \tau+1$ and $b \longrightarrow b+1$. Consequently, the function

$$
E(r, \tau ; b ; m)=2^{2 i \tilde{m}}(1+b)^{2 i m}\left((\tau+b+2)^{2}-r^{2}\right)^{-i m} F\left(i m, i m ; 1 ; \frac{(\tau-b)^{2}-r^{2}}{(\tau+b+2)^{2}-r^{2}}\right)
$$

solves equation (29). Theorem is proved.

Lemma 5.2 We have

$$
\begin{aligned}
& \lim _{\tau \rightarrow 0} K_{1}(\tau, \tau ; m)=1, \\
& \lim _{\tau \rightarrow 0}\left(K_{0}(\tau, \tau ; m)+2 i m K_{1}(\tau, \tau ; m)\right)=i m .
\end{aligned}
$$

The proof of the lemma is omitted. The next proposition is an analog of Propositions 2.9, 2.13, 2.15 [35] and we skip its proof.

Proposition 5.1 The following hold for the kernel functions

$$
\begin{aligned}
& E(0, \tau ; \tau ; m)=1, \\
& E_{r}(\tau-b, \tau ; b ; m)+E_{\tau}(\tau-b, \tau ; b ; m)+\frac{i m}{\tau+1} E(\tau-b, \tau ; b ; m)=0, \\
& K_{1 r}(\tau, \tau ; m)+K_{1 \tau}(\tau, \tau ; m)+\frac{i m}{\tau+1} K_{1}(\tau, \tau ; m)=0, \\
& 2 K_{0 r}(\tau, \tau ; m)+2 K_{0 \tau}(\tau, \tau ; m)+\frac{2 i m}{\tau+1} K_{0}(\tau, \tau ; m)=-m(m+i)(\tau+1)^{-2-i m} .
\end{aligned}
$$

\subsection{Solution with $f, \varphi_{1}, \varphi_{0}$ for equation in the proper time}

Theorem 5.3 Let the function $v=v_{f}(x, r ; b) \in C_{x, r, b}^{d, 2,0}$ be a solution to the Cauchy problem

$$
\left\{\begin{array}{l}
v_{r r}(x, r ; b)-A\left(x, \partial_{x}\right) v(x, r ; b)=0, \\
v(x, 0 ; b)=f(x, b), \quad v_{r}(x, 0 ; b)=0,
\end{array}\right.
$$

while $v=v_{\varphi}(x, r) \in C_{x, r}^{d, 2}$ is a solution to the problem

$$
\left\{\begin{array}{l}
v_{r r}(x, r)-A\left(x, \partial_{x}\right) v(x, r)=0, \\
v(x, 0)=\varphi(x), \quad v_{r}(x, 0)=0 .
\end{array}\right.
$$

Then the function

$$
\begin{aligned}
u(x, \tau)= & \int_{0}^{\tau} d b \int_{0}^{\tau-b} E(r, \tau ; b ; m) v_{f}(x, r ; b) d r+\int_{0}^{\tau} K_{1}(r, \tau ; m) v_{\varphi_{1}}(x, r) d r \\
& +(1+\tau)^{-i m} v_{\varphi_{0}}(x, \tau)+\int_{0}^{\tau}\left[K_{0}(r, \tau ; m)+2 i m K_{1}(r, \tau ; m)\right] v_{\varphi_{0}}(x, r) d r
\end{aligned}
$$

solves the Cauchy problem

$$
\left\{\begin{array}{l}
\partial_{\tau}^{2} u(x, \tau)-A\left(x, \partial_{x}\right) u(x, \tau)+\frac{2 i m}{\tau+1} u_{\tau}(x, \tau)=f(\tau, x) \\
u(x, 0)=\varphi_{0}(x), \quad u_{\tau}(x, 0)=\varphi_{1}(x)
\end{array}\right.
$$


We stress here that the operator $A\left(x, \partial_{x}\right)$ is a pseudo-differential operator without any restriction on its order $d$ or type. Thus, there is no any assumption on the type of equation (28). Furthermore, the source term and initial data may be chosen from the Sobolev spaces as well.

If we assume that $A\left(x, \partial_{x}\right)$ is an elliptic operator of the second order, then equation (28) is strictly hyperbolic. Therefore, from the last theorem and from the well-posedness of the Cauchy problem for the hyperbolic operator in the domain $t>0$ follow the next statements about the fundamental solutions.

Theorem 5.4 Let $\mathcal{E}_{A}$ be a fundamental solution of the Cauchy problem

$$
\left\{\begin{array}{l}
\partial_{r}^{2} \mathcal{E}_{A}\left(x, r ; x_{0}\right)-A\left(x, \partial_{x}\right) \mathcal{E}_{A}\left(x, r ; x_{0}\right)=0 \\
\mathcal{E}_{A}\left(x, 0 ; x_{0}\right)=\delta\left(x-x_{0}\right), \quad \partial_{r} \mathcal{E}_{A}\left(x, 0 ; x_{0}\right)=0
\end{array}\right.
$$

then the distribution with the support in $\left\{(x, \tau) \mid x \in \mathbb{R}^{n}, \tau \geq \tau_{0}\right\}$ that is defined by

$$
\mathcal{E}_{+}\left(x, \tau ; x_{0}, \tau_{0}\right)=\int_{0}^{\tau-\tau_{0}} E\left(r, \tau ; \tau_{0} ; m\right) \mathcal{E}_{A}\left(x, r ; x_{0}\right) d r \quad \text { if } \quad \tau>\tau_{0}>0,
$$

solves the equation

$$
\partial_{\tau}^{2} \mathcal{E}_{+}\left(x, \tau ; x_{0}, \tau_{0}\right)-A\left(x, \partial_{x}\right) \mathcal{E}_{+}\left(x, \tau ; x_{0}, \tau_{0}\right)+\frac{2 i m}{\tau+1} \partial_{\tau} \mathcal{E}_{+}\left(x, \tau ; x_{0}, \tau_{0}\right)=\delta\left(x-x_{0}\right) \delta\left(\tau-\tau_{0}\right) .
$$

Theorem 5.5 Let $\mathcal{E}_{A}$ be a fundamental solution of the Cauchy problem, that is

$$
\left\{\begin{array}{l}
\partial_{r}^{2} \mathcal{E}_{A}\left(x, r ; x_{0}\right)-A\left(x, \partial_{x}\right) \mathcal{E}_{A}\left(x, r ; x_{0}\right)=0 \\
\mathcal{E}_{A}\left(x, 0 ; x_{0}\right)=\delta\left(x-x_{0}\right), \quad \partial_{r} \mathcal{E}_{A}\left(x, 0 ; x_{0}\right)=0
\end{array}\right.
$$

then the distributions

$$
\begin{aligned}
& \mathcal{E}_{0}\left(x, \tau ; x_{0}\right)=(1+\tau)^{-i m} \mathcal{E}_{A}\left(x, \tau ; x_{0}\right)+\int_{0}^{\tau}\left[K_{0}(r, \tau ; m)+2 i m K_{1}(r, \tau ; m)\right] \mathcal{E}_{A}\left(x, r ; x_{0}\right) d r, \\
& \mathcal{E}_{1}\left(x, \tau ; x_{0}\right)=\int_{0}^{\tau} K_{1}(r, \tau ; m) \mathcal{E}_{A}\left(x, r ; x_{0}\right) d r
\end{aligned}
$$

solve the Cauchy problem

$$
\left\{\begin{array}{l}
\partial_{\tau}^{2} \mathcal{E}_{i}\left(x, \tau ; x_{0}\right)-A\left(x, \partial_{x}\right) \mathcal{E}_{i}\left(x, \tau ; x_{0}\right)+\frac{2 i m}{\tau+1} \partial_{\tau} \mathcal{E}_{i}\left(x, \tau ; x_{0}\right)=0, \\
\mathcal{E}_{i}\left(x, 0 ; x_{0}\right)=\delta_{i 0} \delta\left(x-x_{0}\right), \quad \partial_{\tau} \mathcal{E}_{i}\left(x, 0 ; x_{0}\right)=\delta_{i 1} \delta\left(x-x_{0}\right), \quad i=0,1
\end{array}\right.
$$

\subsection{Proof of Theorem 5.3 for problem with $\varphi_{0}$. Case of $f=0, \varphi_{1}=0$}

Next, we will suppress subscript $\varphi_{0}$ of $v_{\varphi_{0}}(x, \tau)$. It is convenient to split the solution $u(x, \tau)$ into two parts $u(x, \tau)=\widetilde{u}(x, \tau)+\widetilde{\widetilde{u}}(x, \tau)$, where

$$
\begin{aligned}
& \widetilde{u}(x, \tau)=(1+\tau)^{-i m} v(x, \tau)+\int_{0}^{\tau} K_{0}(r, \tau ; m) v(x, r) d r, \\
& \widetilde{\widetilde{u}}(x, \tau)=2 i m \int_{0}^{\tau} K_{1}(r, \tau ; m) v(x, r) d r .
\end{aligned}
$$

It is easy to see that $u(x, 0)=\varphi_{0}(x)$ and

$$
\begin{aligned}
u_{\tau}(x, \tau)= & \partial_{\tau}\left((1+\tau)^{-i m} v(x, \tau)\right)+\partial_{\tau} \int_{0}^{\tau}\left[K_{0}(r, \tau ; m)+2 i m K_{1}(r, \tau ; m)\right] v(x, r) d r \\
= & -i m(1+\tau)^{-i m-1} v(x, \tau)+(1+\tau)^{-i m} \partial_{\tau} v(x, \tau) \\
& +\left[K_{0}(\tau, \tau ; m)+2 i m K_{1}(\tau, \tau ; m)\right] v(x, \tau) \\
& +\int_{0}^{\tau}\left[\partial_{\tau} K_{0}(r, \tau ; m)+2 i m \partial_{\tau} K_{1}(r, \tau ; m)\right] v(x, r) d r
\end{aligned}
$$


It follows from Lemma 5.2 that

$$
\lim _{\tau \rightarrow 0} u_{\tau}(x, \tau)=-\lim _{\tau \rightarrow 0} i m v(x, 0)+\lim _{\tau \rightarrow 0}\left[K_{0}(\tau, \tau ; m)+2 i m K_{1}(\tau, \tau ; m)\right] v(x, 0)=0
$$

According to Theorem 5.3 the function $\widetilde{\widetilde{u}}(x, \tau)(33)$ solves the equation, therefore it remains to verify that the function $\widetilde{u}(x, \tau)(32)$ solves the equation. For the first order derivative we obtain

$$
\begin{aligned}
\widetilde{u}_{\tau}(x, \tau)= & \partial_{\tau}\left((1+\tau)^{-i m} v(x, \tau)\right)+\partial_{\tau} \int_{0}^{\tau} K_{0}(r, \tau ; m) v(x, r) d r \\
= & -i m(1+\tau)^{-i m-1} v(x, \tau)+(1+\tau)^{-i m} \partial_{\tau} v(x, \tau) \\
& +K_{0}(\tau, \tau ; m) v(x, \tau)+\int_{0}^{\tau} K_{0 \tau}(r, \tau ; m) v(x, r) d r
\end{aligned}
$$

For the second order derivative we have

$$
\begin{aligned}
\widetilde{u}_{\tau \tau}(x, \tau)= & -i m(-i m-1)(1+\tau)^{-i m-2} v(x, \tau)-2 i m(1+\tau)^{-i m-1} v_{\tau}(x, \tau)+(1+\tau)^{-i m} v_{\tau \tau}(x, \tau) \\
& +\partial_{\tau}\left\{K_{0}(\tau, \tau ; m) v(x, \tau)+\int_{0}^{\tau} K_{0 \tau}(r, \tau ; m) v(x, r) d r\right\} .
\end{aligned}
$$

Thus,

$$
\begin{aligned}
\widetilde{u}_{\tau \tau}(x, \tau)= & -i m(-i m-1)(1+\tau)^{-i m-2} v(x, \tau)-2 i m(1+\tau)^{-i m-1} v_{\tau}(x, \tau)+(1+\tau)^{-i m} v_{\tau \tau}(x, \tau) \\
& +\left[K_{0 r}(\tau, \tau ; m) v(x, \tau)+2 K_{0 \tau}(\tau, \tau ; m) v(x, \tau)+K_{0}(\tau, \tau ; m) v_{\tau}(x, \tau)\right. \\
& \left.+\int_{0}^{\tau} K_{0 \tau \tau}(r, \tau ; m) v(x, r) d r\right]
\end{aligned}
$$

Next we consider an application of the operator $A\left(x, \partial_{x}\right)$ to the function $(32)$ :

$$
\begin{aligned}
A\left(x, \partial_{x}\right) \widetilde{u}(x, \tau) & =A\left(x, \partial_{x}\right)(1+\tau)^{-i m} v(x, \tau)+\int_{0}^{\tau} K_{0}(r, \tau ; m) A\left(x, \partial_{x}\right) v(x, r) d r \\
& =(1+\tau)^{-i m} A\left(x, \partial_{x}\right) v(x, \tau)+B(x, \tau)
\end{aligned}
$$

where we have denoted

$$
B(x, \tau):=\int_{0}^{\tau} K_{0}(r, \tau ; m) v_{r r}(x, r) d r .
$$

Integrating by parts and using $K_{0}(0, \tau ; m)=0$, we obtain

$$
\begin{aligned}
B(x, \tau)= & K_{0}(\tau, \tau ; m) v_{r}(x, \tau)-\int_{0}^{\tau} K_{0 r}(r, \tau ; m) v_{r}(x, r) d r \\
= & K_{0}(\tau, \tau ; m) v_{r}(x, \tau)-K_{0 r}(\tau, \tau ; m) v(x, \tau)+K_{0 r}(0, \tau ; m) v(x, 0) \\
& +\int_{0}^{\tau} K_{0 r r}(r, \tau ; m) v(x, r) d r .
\end{aligned}
$$

Since $K_{0 r}(0, \tau ; m)=0$, it follows

$$
\begin{aligned}
A\left(x, \partial_{x}\right) \widetilde{u}(x, \tau)= & (1+\tau)^{-i m} A\left(x, \partial_{x}\right) v(x, \tau)+\left[K_{0}(\tau, \tau ; m) v_{r}(x, \tau)-K_{0 r}(\tau, \tau ; m) v(x, \tau)\right. \\
& \left.+K_{0 r}(0, \tau ; m) v(x, 0)+\int_{0}^{\tau} K_{0 r r}(r, \tau ; m) v(x, r) d r\right]
\end{aligned}
$$


Thus,

$$
\begin{aligned}
& \widetilde{u}_{\tau \tau}(x, \tau)-A\left(x, \partial_{x}\right) \widetilde{u}(x, \tau)+\frac{2 i m}{\tau+1} \widetilde{u}_{\tau}(x, \tau) \\
= & \left\{-i m(-i m-1)(1+\tau)^{-i m-2} v(x, \tau)-2 i m(1+\tau)^{-i m-1} v_{\tau}(x, \tau)+(1+\tau)^{-i m} v_{\tau \tau}(x, \tau)\right. \\
& +\left[K_{0 r}(\tau, \tau ; m) v(x, \tau)+2 K_{0 \tau}(\tau, \tau ; m) v(x, \tau)+K_{0}(\tau, \tau ; m) v_{\tau}(x, \tau)\right. \\
& \left.\left.+\int_{0}^{\tau} K_{0 \tau \tau}(r, \tau ; m) v(x, r) d r\right]\right\}-\left\{(1+\tau)^{-i m} A\left(x, \partial_{x}\right) v(x, \tau)\right. \\
& \left.+\left[K_{0}(\tau, \tau ; m) v_{r}(x, \tau)-K_{0 r}(\tau, \tau ; m) v(x, \tau)+\int_{0}^{\tau} K_{0 r r}(r, \tau ; m) v(x, r) d r\right]\right\} \\
& +\frac{2 i m}{\tau+1}\left\{-i m(1+\tau)^{-i m-1} v(x, \tau)+(1+\tau)^{-i m} v_{\tau}(x, \tau)\right. \\
& \left.+K_{0}(\tau, \tau ; m) v(x, \tau)+\int_{0}^{\tau} K_{0 \tau}(r, \tau ; m) v(x, r) d r\right\} .
\end{aligned}
$$

By application of (30) and the definition of the function $v=v(x, \tau)$ we obtain

$$
\begin{aligned}
& \widetilde{u}_{\tau \tau}(x, \tau)-A\left(x, \partial_{x}\right) \widetilde{u}(x, \tau)+\frac{2 i m}{\tau+1} \widetilde{u}_{\tau}(x, \tau) \\
= & {\left[-i m(-i m-1)(1+\tau)^{-i m-2}+2 K_{0 r}(\tau, \tau ; m)+2 K_{0 \tau}(\tau, \tau ; m)\right.} \\
& \left.+\frac{2 i m}{\tau+1}\left\{-i m(1+\tau)^{-i m-1}+K_{0}(\tau, \tau ; m)\right\}\right] v(x, \tau) \\
= & m(m+i m)(1+\tau)^{-i m-2} v(x, \tau) \\
& +\left[2 K_{0 r}(\tau, \tau ; m) v(x, \tau)+2 K_{0 \tau}(\tau, \tau ; m)+\frac{2 i m}{\tau+1} K_{0}(\tau, \tau ; m)\right] v(x, \tau) .
\end{aligned}
$$

Then we apply the last equation of Proposition 5.1 and obtain

$$
m(m+i m)(1+\tau)^{-i m-2}+\left[2 K_{0 r}(\tau, \tau ; m)+2 K_{0 \tau}(\tau, \tau ; m)+\frac{2 i m}{\tau+1} K_{0}(\tau, \tau ; m)\right]=0 .
$$

Thus, this case of Theorem 5.3 is proved.

\subsection{Proof of Theorem 5.3 for problem with $\varphi_{1}$. Case of $f=0, \varphi_{0}=0$}

Next, we will suppress subscript $\varphi_{1}$ of $v_{\varphi_{1}}(x, \tau)$. It is evident that $u(x, 0)=0$. For the derivative $\partial_{\tau} u(x, \tau)$ we have

$$
\partial_{\tau} u(x, \tau)=\partial_{\tau} \int_{0}^{\tau} K_{1}(r, \tau ; m) v(x, r) d r=K_{1}(\tau, \tau ; m) v(x, \tau)+\int_{0}^{\tau} K_{1 \tau}(r, \tau ; m) v(x, r) d r .
$$

In particular,

$$
\lim _{\tau \rightarrow 0} \partial_{\tau} u(x, \tau)=\lim _{\tau \rightarrow 0} K_{1}(\tau, \tau ; m) v(x, \tau)=\varphi_{1}(x)
$$

where we have used

$$
K_{1}(\tau, \tau ; m)=2^{2 i m}\left((\tau+2)^{2}-\tau^{2}\right)^{-i m}
$$


Then we use (34) to find the second order derivative

$$
\partial_{\tau}^{2} u(x, \tau)=K_{1 r}(\tau, \tau ; m) v(x, \tau)+2 K_{1 \tau}(\tau, \tau ; m) v(x, \tau)+K_{1}(\tau, \tau ; m) v_{\tau}(x, \tau)+\int_{0}^{\tau} K_{1 \tau \tau}(r, \tau ; m) v(x, r) d r .
$$

Using the definition of the function $v(x, r)$ and the integration by parts twice, we derive

$$
\begin{aligned}
A\left(x, \partial_{x}\right) u(x, \tau)= & \int_{0}^{\tau} K_{1}(r, \tau ; m) A\left(x, \partial_{x}\right) v(x, r) d r \\
= & \int_{0}^{\tau} K_{1}(r, \tau ; m) v_{r r}(x, r) d r \\
= & K_{1}(\tau, \tau ; m) v_{r}(x, \tau)-\left\{K_{1 r}(\tau, \tau ; m) v(x, \tau)-K_{1 r}(0, \tau ; m) v(x, 0)\right. \\
& \left.-\int_{0}^{\tau} K_{1 r r}(r, \tau ; m) v(x, r) d r\right\} .
\end{aligned}
$$

Since $K_{1 r}(0, \tau ; m)=0$, we obtain

$$
A\left(x, \partial_{x}\right) u(x, \tau)=K_{1}(\tau, \tau ; m) v_{r}(x, \tau)-K_{1 r}(\tau, \tau ; m) v(x, \tau)+\int_{0}^{\tau} K_{1 r r}(r, \tau ; m) v(x, r) d r .
$$

Consequently, according to (31) of Theorem 5.1 and Proposition 5.1 since $v_{r}(x, \tau)=v_{\tau}(x, \tau)$, we obtain

$$
\begin{aligned}
& u_{\tau \tau}(x, \tau)-A\left(x, \partial_{x}\right) u(x, \tau)+\frac{2 i m}{\tau+1} u_{\tau}(x, \tau) \\
= & \left\{K_{1 r}(\tau, \tau ; m) v(x, \tau)+K_{1}(\tau, \tau ; m) v_{\tau}(x, \tau)+2 K_{1 \tau}(\tau, \tau ; m) v(x, \tau)+\int_{0}^{\tau} K_{1 \tau \tau}(r, \tau ; m) v(x, r) d r\right\} \\
& -\left\{K_{1}(\tau, \tau ; m) v_{r}(x, \tau)-K_{1 r}(\tau, \tau ; m) v(x, \tau)+\int_{0}^{\tau} K_{1 r r}(r, \tau ; m) v(x, r) d r\right\} \\
& +\frac{2 i m}{\tau+1}\left\{K_{1}(\tau, \tau ; m) v(x, \tau)+\int_{0}^{\tau} K_{1 \tau}(r, \tau ; m) v(x, r) d r\right\} \\
= & \left\{2 K_{1 r}(\tau, \tau ; m)+2 K_{1 \tau}(\tau, \tau ; m)+\frac{2 i m}{\tau+1} K_{1}(\tau, \tau ; m)\right\} v(x, \tau)=0 .
\end{aligned}
$$

Thus, this case of Theorem 5.3 is proved.

\subsection{Proof of Theorem 5.3 for problem with $f$. Case of $\varphi_{0}=\varphi_{1}=0$}

Next, we will suppress subscript $f$ of $v_{f}(x, r ; b)$. We write $v(x, r ; b)$ for $v_{f}(x, r ; b)$ and calculate the derivative

$$
\begin{aligned}
\partial_{\tau} u(x, \tau) & =\int_{0}^{\tau} d b \partial_{\tau} \int_{0}^{\tau-b} E(r, \tau ; b ; m) v(x, r ; b) d r \\
& =\int_{0}^{\tau} E(\tau-b, \tau ; b ; m) v(x, \tau-b ; b) d b+\int_{0}^{\tau} d b \int_{0}^{\tau-b} E_{\tau}(r, \tau ; b ; m) v(x, r ; b) d r .
\end{aligned}
$$

It follows that the function takes required initial data as well as

$$
\begin{aligned}
\partial_{\tau}^{2} u(x, \tau)= & E(0, \tau ; \tau ; m) f(x, \tau)+\int_{0}^{\tau} E_{r}(\tau-b, \tau ; b ; m) v(x, \tau-b ; b) d b \\
& +\int_{0}^{\tau} E(\tau-b, \tau ; b ; m) v_{\tau}(x, \tau-b ; b) d b+2 \int_{0}^{\tau} E_{\tau}(\tau-b, \tau ; b ; m) v(x, \tau-b ; b) d b \\
& +\int_{0}^{\tau} d b \int_{0}^{\tau-b} \partial_{\tau}^{2} E(r, \tau ; b ; m) v(x, r ; b) d r .
\end{aligned}
$$


Then using the definition of the function $v=v(x, r)$ and the integration by parts twice, we derive

$$
\begin{aligned}
A\left(x, \partial_{x}\right) u(x, \tau) & =\int_{0}^{\tau} d b \int_{0}^{\tau-b} E(r, \tau ; b ; m) A\left(x, \partial_{x}\right) v(x, r ; b) d r \\
& =\int_{0}^{\tau} d b \int_{0}^{\tau-b} E(r, \tau ; b ; m) \partial_{r}^{2} v(x, r ; b) d r \\
& =\int_{0}^{\tau} d b\left[E(\tau-b, \tau ; b ; m) v_{r}(x, \tau-b ; b)-E(0, \tau ; b ; m) v_{r}(x, 0 ; b)\right. \\
& \left.-\int_{0}^{\tau-b} \partial_{r} E(r, \tau ; b ; m) v_{r}(x, r ; b) d r\right] \\
& =\int_{0}^{\tau} d b\left[E(\tau-b, \tau ; b ; m) v_{r}(x, \tau-b ; b)-\int_{0}^{\tau-b} \partial_{r} E(r, \tau ; b ; m) v_{r}(x, r ; b) d r\right]
\end{aligned}
$$

Hence

$$
\begin{aligned}
& u_{\tau \tau}(x, \tau)-A\left(x, \partial_{x}\right) u(x, \tau)+\frac{2 i m}{\tau+1} u_{\tau}(x, \tau) \\
= & {\left[E(0, \tau ; \tau ; m) f(x, \tau)+\int_{0}^{\tau} E_{r}(\tau-b, \tau ; b ; m) v(x, \tau-b ; b) d b\right.} \\
& +\int_{0}^{\tau} E(\tau-b, \tau ; b ; m) v_{\tau}(x, \tau-b ; b) d b+2 \int_{0}^{\tau} E_{\tau}(\tau-b, \tau ; b ; m) v(x, \tau-b ; b) d b \\
& \left.+\int_{0}^{\tau} d b \int_{0}^{\tau-b} \partial_{\tau}^{2} E(r, \tau ; b ; m) v(x, r ; b) d r\right] \\
& -\left[\int_{0}^{\tau} d b\left\{E(\tau-b, \tau ; b ; m) v_{r}(x, \tau-b ; b)-\int_{0}^{\tau-b} \partial_{r} E(r, \tau ; b ; m) v_{r}(x, r ; b) d r\right\}\right] \\
& +\frac{2 i m}{\tau+1}\left[\int_{0}^{\tau} E(\tau-b, \tau ; b ; m) v(x, \tau-b ; b) d b+\int_{0}^{\tau} d b \int_{0}^{\tau-b} E_{\tau}(r, \tau ; b ; m) v(x, r ; b) d r\right] .
\end{aligned}
$$

On the other hand, the integration by parts leads to

$$
\begin{aligned}
& \int_{0}^{\tau} d b \int_{0}^{\tau-b} E_{r}(r, \tau ; b ; m) v_{r}(x, r ; b) d r \\
= & \int_{0}^{\tau}\left\{E_{r}(\tau-b, \tau ; b ; m) v(x, \tau-b ; b)-E_{r}(0, \tau ; b ; m) v(x, 0 ; b)-\int_{0}^{\tau-b} E_{r r}(r, \tau ; b ; m) v(x, r ; b) d r\right\} d b .
\end{aligned}
$$

Then we use $E_{r}(0, \tau ; b ; m)=0$. Consequently,

$$
\begin{aligned}
& \int_{0}^{\tau} d b \int_{0}^{\tau-b} \partial_{r} E(r, \tau ; b ; m) v_{r}(x, r ; b) d r \\
= & \int_{0}^{\tau}\left\{\partial_{r} E(\tau-b, \tau ; b ; m) v(x, \tau-b ; b)-\partial_{r} E(0, \tau ; b ; m) f(x, b)-\int_{0}^{\tau-b} \partial_{r r} E(r, \tau ; b ; m) v(x, r ; b) d r\right\} d b \\
= & \int_{0}^{\tau} E_{r}(\tau-b, \tau ; b ; m) v(x, \tau-b ; b) d b-\int_{0}^{\tau} d b \int_{0}^{\tau-b} E_{r r}(r, \tau ; b ; m) v(x, r ; b) d r
\end{aligned}
$$

Thus,

$$
u_{\tau \tau}(x, \tau)-A\left(x, \partial_{x}\right) u(x, \tau)+\frac{2 i m}{\tau+1} u_{\tau}(x, \tau)
$$




$$
\begin{aligned}
= & {\left[E(0, \tau ; \tau ; m) f(x, \tau)+\int_{0}^{\tau} E_{r}(\tau-b, \tau ; b ; m) v(x, \tau-b ; b) d b\right.} \\
& +\int_{0}^{\tau} E(\tau-b, \tau ; b ; m) v_{\tau}(x, \tau-b ; b) d b+2 \int_{0}^{\tau} E_{\tau}(\tau-b, \tau ; b ; m) v(x, \tau-b ; b) d b \\
& \left.+\int_{0}^{\tau} d b \int_{0}^{\tau-b} \partial_{\tau}^{2} E(r, \tau ; b ; m) v(x, r ; b) d r\right]-\int_{0}^{\tau} E(\tau-b, \tau ; b ; m) v_{r}(x, \tau-b ; b) d b \\
& +\left\{\int_{0}^{\tau} E_{r}(\tau-b, \tau ; b ; m) v(x, \tau-b ; b) d b-\int_{0}^{\tau} d b \int_{0}^{\tau-b} E_{r r}(r, \tau ; b ; m) v(x, r ; b) d r\right\} \\
& +\frac{2 i m}{\tau+1}\left[\int_{0}^{\tau} E(\tau-b, \tau ; b ; m) v(x, \tau-b ; b) d b+\int_{0}^{\tau} d b \int_{0}^{\tau-b} E_{\tau}(r, \tau ; b ; m) v(x, r ; b) d r\right] .
\end{aligned}
$$

The double integrals of the last equation can be unified as follows

$$
\begin{aligned}
& \int_{0}^{\tau} d b \int_{0}^{\tau-b} \partial_{\tau}^{2} E(r, \tau ; b ; m) v(x, r ; b) d r-\int_{0}^{\tau} d b \int_{0}^{\tau-b} E_{r r}(r, \tau ; b ; m) v(x, r ; b) d r \\
& +\frac{2 i m}{\tau+1} \int_{0}^{\tau} d b \int_{0}^{\tau-b} E_{\tau}(r, \tau ; b ; m) v(x, r ; b) d r \\
= & \int_{0}^{\tau} d b \int_{0}^{\tau-b}\left\{E_{\tau \tau}(r, \tau ; b ; m)-E_{r r}(r, \tau ; b ; m)+\frac{2 i m}{\tau+1} E_{\tau}(r, \tau ; b ; m)\right\} v(x, r ; b) d r=0 .
\end{aligned}
$$

Hence, according to (29) we derive

$$
\begin{aligned}
& u_{\tau \tau}(x, \tau)-A\left(x, \partial_{x}\right) u(x, \tau)+\frac{2 i m}{\tau+1} u_{\tau}(x, \tau) \\
= & E(0, \tau ; \tau ; m) f(x, \tau)+\int_{0}^{\tau} E_{r}(\tau-b, \tau ; b ; m) v(x, \tau-b ; b) d b \\
& +\int_{0}^{\tau} E(\tau-b, \tau ; b ; m) v_{\tau}(x, \tau-b ; b) d b+2 \int_{0}^{\tau} E_{\tau}(\tau-b, \tau ; b ; m) v(x, \tau-b ; b) d b \\
& -\int_{0}^{\tau} E(\tau-b, \tau ; b ; m) v_{r}(x, \tau-b ; b) d b+\int_{0}^{\tau} E_{r}(\tau-b, \tau ; b ; m) v(x, \tau-b ; b) d b \\
& +\frac{2 i m}{\tau+1} \int_{0}^{\tau} E(\tau-b, \tau ; b ; m) v(x, \tau-b ; b) d b .
\end{aligned}
$$

Next we use $v_{\tau}(x, \tau-b ; b)=v_{r}(x, \tau-b ; b)$ in

$$
\int_{0}^{\tau} E(\tau-b, \tau ; b ; m) v_{\tau}(x, \tau-b ; b) d b-\int_{0}^{\tau} E(\tau-b, \tau ; b ; m) v_{r}(x, \tau-b ; b) d b=0 .
$$

Hence

$$
\begin{aligned}
& u_{\tau \tau}(x, \tau)-A\left(x, \partial_{x}\right) u(x, \tau)+\frac{2 i m}{\tau+1} u_{\tau}(x, \tau) \\
= & E(0, \tau ; \tau ; m) f(x, \tau) \\
& +\int_{0}^{\tau} E_{r}(\tau-b, \tau ; b ; m) v(x, \tau-b ; b) d b+2 \int_{0}^{\tau} E_{\tau}(\tau-b, \tau ; b ; m) v(x, \tau-b ; b) d b \\
& +\int_{0}^{\tau} E_{r}(\tau-b, \tau ; b ; m) v(x, \tau-b ; b) d b+\frac{2 i m}{\tau+1} \int_{0}^{\tau} E(\tau-b, \tau ; b ; m) v(x, \tau-b ; b) d b \\
= & E(0, \tau ; \tau ; m) f(x, \tau) \\
& +\int_{0}^{\tau}\left[2 E_{r}(\tau-b, \tau ; b ; m)+2 E_{\tau}(\tau-b, \tau ; b ; m)+\frac{2 i m}{\tau+1} E(\tau-b, \tau ; b ; m)\right] v(x, \tau-b ; b) d b .
\end{aligned}
$$


Then we apply Proposition 5.1 and obtain

$$
u_{\tau \tau}(x, \tau)-A\left(x, \partial_{x}\right) u(x, \tau)+\frac{2 i m}{\tau+1} u_{\tau}(x, \tau)=f(x, \tau) .
$$

This case of Theorem 5.3 is proved. Theorem 5.3 is proved.

\section{References}

[1] A.O. Barut, I.H. Duru, Exact solutions of the Dirac equation in spatially flat Robertson-Walker spacetimes, Phys. Rev. D (3) 36, no. 12 (1987) 3705-3711.

[2] H. Bateman, A. Erdelyi, Higher Transcendental Functions, Vol. 1,2, McGraw-Hill, New York, 1954.

[3] N.D. Birrell, P.C.W. Davies, Quantum Fields in Curved Space, Cambridge, New York, Cambridge University Press, 1984.

[4] A. V. Bitsadze, Some Classes of Partial Differential Equations. Advanced Studies in Contemporary Mathematics 4, Gordon and Breach Science Publishers, New York, 1988.

[5] N.N. Bogolyubov, D.V. Shirkov, Introduction to Quantum Field Theory, Fourth ed., Nauka, Moscow, 1984.

[6] D.R. Brill, J.A. Wheeler, Interaction of Neutrinos and Gravitational Fields, Rev. Mod. Phys. 29 (1957) 465-479.

[7] S. Carroll, Spacetime and Geometry. An Introduction to General Relativity, Addison Wesley, San Francisco, CA, 2004.

[8] R. W. Carroll, R. E. Showalter, Singular and degenerate Cauchy problems, Mathematics in Science and Engineering, Vol. 127, Academic Press [Harcourt Brace Jovanovich, Publishers], New York-London, 1976.

[9] S. Delache, J. Leray, Calcul de la solution élémentaire de l'opérateur d'Euler-Poisson-Darboux et de l'opérateur de Tricomi-Clairaut, hyperbolique, d'ordre 2, Bull. Soc. Math. France 99 (1971) 313-336.

[10] J. B. Diaz, H. F. Weinberger, A solution of the singular initial value problem for the Euler-PoissonDarboux equation, Proc. Amer. Math. Soc. 4 (1953) 703-715.

[11] A. Einstein, W. de Sitter, On the relation between the expansion and the mean density of the universe, Proc. Natn. Acad. Sci. U.S.A. 18 (1932) 213-214.

[12] F. Finster, M. Reintjes, The Dirac equation and the normalization of its solutions in a closed FriedmannRobertson-Walker universe, Classical Quantum Gravity 26, no. 10(2009) 105021, 20 pp.

[13] O. Gron, S. Hervik, Einstein's General Theory of Relativity: with Modern Applications in Cosmology, Springer-Verlag, New York, LLC, 2007.

[14] M. Hamouda, M. A. Hamza, A. Palmieri, A note on the nonexistence of global solutions to the semilinear wave equation with nonlinearity of derivative-type in the generalized Einstein-de Sitter spacetime, Commun. Pure Appl. Anal., in press, doi:10.3934/cpaa.2021127.

[15] J. C. Huang, N. O. Santos; A. Kleber, Neutrinos in a Robertson-Walker universe. Classical Quantum Gravity 12, no. 5 (1995) 1245-1257.

[16] X.-B. Huang, Exact solutions of the Dirac equation in Robertson-Walker space-time, ArXiv:grqc/0501077

[17] X.-B. Huang, Neutrino oscillations in de Sitter space-time, ArXiv:hep-th/0502165 
[18] C. Møller, The Theory of Relativity, Oxford, Clarendon Press, 1952.

[19] H. Ohanian, R. Ruffini, Gravitation and Spacetime, Norton, New York, 1994.

[20] M. D. de Oliveira, A.G.M. Schmidt, Exact solutions of Dirac equation on a static curved space-time, Ann. Physics 401 (2019) 21-39.

[21] A. Palmieri, Integral representation formula for the solution of a wave equation with timedependent damping and mass in the scale-invariant case, Math. Methods Appl. Sci., in press, https://doi.org/10.1002/mma.7603.

[22] C. Parenti, H. Tahara, Asymptotic expansions of distribution solutions of some Fuchsian hyperbolic equations, Publ. Res. Inst. Math. Sci. 23, no. 6 (1987) 909-922.

[23] L. E. Parker, D. J. Toms, Quantum Field Theory in Curved Spacetime, Quantized fields and gravity, Cambridge Monographs on Mathematical Physics, Cambridge University Press, Cambridge, 2009.

[24] P. Schluter, K.-H. Wietschorke, W. Greiner, The Dirac equation in orthogonal coordinate systems. I. The local representation, J. Phys. A: Math. Gen. 16 (1983) 1999-2016.

[25] E. Schrödinger, Diracsches Elektron im Schwerefeld, Preuss. Akad. Wiss. Berlin, Ber. 11-12 (1932) 105-128.

[26] E. L. Shishkina, S. M. Sitnik, General form of the Euler-Poisson-Darboux equation and application of the transmutation method, Electron. J. Differential Equations 2017, Paper No. 177, 20 pp.

[27] V. M. Villalba, U. Percoco, Separation of variables and exact solution to Dirac and Weyl equations in Robertson-Walker space-times, J. Math. Phys. 31, no. 3 (1990) 715-720.

[28] A. Weinstein, The singular solutions and the Cauchy problem for generalized Tricomi equations, Comm. Pure Appl. Math. 7 (1954) 105-116.

[29] J. Wirth, Solution representations for a wave equation with weak dissipation, Math. Methods Appl. Sci. 27, no. 1 (2004) 101-124.

[30] K. Yagdjian, A Note on the fundamental folution for the Tricomi-type equation in the hyperbolic domain, J. Differential Equations 206 (2004) 227-252.

[31] K. Yagdjian, Integral transform approach to generalized Tricomi equations, J. Differential Equations 259, no. 11 (2015) 5927-5981.

[32] K. Yagdjian, A. Galstian, Fundamental solutions for the Klein-Gordon equation in de Sitter spacetime, Comm. Math. Phys. 285 (2009) 293-344.

[33] K. Yagdjian, Fundamental solutions of the Dirac operator in the Friedmann-Lemaittre-Robertson-Walker spacetime, Ann. Physics 421 (2020) 168266.

[34] K. Yagdjian, Huygens' principle for the generalized Dirac operator in curved spacetime, J. Phys. A: Mathematical and Theoretical, 54 (2021) 095204

[35] K. Yagdjian, Integral transform approach to solving Klein-Gordon equation with variable coefficients, Math. Nachr. 288, no. 17-18 (2015) 2129-2152.

[36] A. Zecca, Solution of the Dirac equation in expanding universes, Internat. J. Theoret. Phys. 45, no. 1 (2006) 47-55. 